\title{
Constraining the past X-ray luminosity of AGN in clusters of galaxies: The role of resonant scattering
}

\author{
S. Yu. Sazonov ${ }^{1,2}$, R. A. Sunyaev ${ }^{1,2}$, and C. K. Cramphorn ${ }^{1}$ \\ 1 Max-Planck-Institut für Astrophysik, Karl-Schwarzschild-Str. 1, 85740 Garching bei München, Germany \\ 2 Space Research Institute, Russian Academy of Sciences, Profsoyuznaya 84/32, 117997 Moscow, Russia
}

\author{
Received 12 April 2002 / Accepted 28 June 2002
}

\begin{abstract}
Only a small fraction of galactic nuclei in the nearby universe are luminous; most of them are currently dim. We investigate the feasibility of constraining the X-ray luminosity in the recent past (up to $\sim 10^{6}$ years ago) of the nucleus of a cluster dominant galaxy by measuring the contribution of scattered radiation from the central source to the surface brightness of the intracluster gas dominated by thermal emission. We show that resonance X-ray lines present an advantage over the adjacent continuum, because the relative contribution of the scattered component is typically larger in the line case by a significant factor of 3-10. As an example, we estimate the level of constraint that could be derived from future fine spectroscopic observations on the past X-ray luminosity of the nearby M 87 and Cygnus A active galaxies. For instance, scattered line radiation should be detectable from the Virgo cluster if the X-ray luminosity of M 87 was a few times $10^{44} \mathrm{erg} \mathrm{s}^{-1}$, or $10^{-3}$ of its Eddington luminosity, until a few times $10^{5}$ years ago. For comparison, upper limits inferred from the available XMM-Newton and Chandra $\mathrm{X}$-ray continuum data are typically 1-2 orders of magnitude weaker.

The same method can be applied to distant powerful quasars (at redshifts $z \gtrsim 1$ ) if they have cluster-like gaseous coronae, as suggested by Rosat and Chandra observations of active galaxies at $z \lessgtr 1$. Their surface brightness profiles in the X-ray continuum above $\sim 10 \mathrm{keV} \gg k T /(1+z)$ (where $T$ is the gas temperature) should be dominated by redshifted scattered radiation from the quasar. Therefore, measurements with forthcoming mirror-optics hard X-ray telescopes could give information on the lifetime of quasars and parameters of the hot gas around them.
\end{abstract}

Key words. galaxies: active - galaxies: quasars: general - scattering - X-rays: galaxies: clusters

\section{Introduction}

It is commonly believed that active galactic nuclei (AGN) are powered by accretion onto a supermassive black hole. However, a detailed description of this process is still missing. One of the principal obstacles to making progress here is that we know very little from observations about the variability of AGN on timescales longer than a few tens of years.

Nuclear variability on the longest timescales up to the Hubble time is expected to be governed by the supply of gas from galactic scales to the central kpc. Such transport can be triggered by mergers and interactions of galaxies (e.g. Hernquist 1989). There are reasons to believe that AGN can be strongly variable on timescales much shorter than the Hubble time. For example, Ciotti \& Ostriker $(1997,2001)$, in a followup to Binney \& Tabor (1995), have suggested a mechanism for elliptical galaxies in which an accreting central black hole heats the ambient gas to the point at which the accretion stops, then fueling resumes after the gas has cooled. In this model, strong outbursts during which the AGN luminosity approaches

Send offprint requests to: S. Yu. Sazonov, e-mail: sazonov@mpa-garching.mpg.de the Eddington critical value can occur every $10^{8}-10^{9}$ years. A similar outburst behaviour but on shorter timescales of typically $10^{6}$ years can result from thermal-viscous instability expected to operate in AGN accretion disks (Lin \& Shield 1986; Mineshige \& Shields 1990; Siemiginiowska et al. 1996; Burderi et al. 1998). We may also mention tidal disruption of stars by supermassive black holes, which can give rise to rare (every $10^{4}-10^{5}$ years per galaxy), short (months to years) and bright (sub-Eddington) flares (Rees 1988).

Since we are unable to explore directly the long-term variability of galactic nuclei, some indirect methods are in need. A feasible way would be to observe radiation which was emitted in the past by a galactic nucleus and later scattered toward us by the interstellar medium. Since the characteristic size of a galaxy is a few tens kpc, we could see the "echo" of previous AGN activity $\sim 10^{5}$ years after the central source turned off. This idea has been put forward by Sunyaev et al. (1993) in relation to our Galaxy. It was suggested that the diffuse, hard X-ray emission observed today in the direction of giant molecular complexes in the central $100 \mathrm{pc}$ of the Milky Way is scattered radiation emitted by Sgr A* in the past. This hypothesis has been 
confirmed by the detection with the ASCA and Chandra satellites of diffuse emission in the $6.4 \mathrm{keV}$ fluorescence iron line from the Sgr B2 cloud (Koyama et al. 1996; Murakami et al. 2001). Cramphorn \& Sunyaev (2002) have recently elaborated on this problem and derived upper limits on the X-ray luminosity of Sgr A* during the past $10^{5}$ years.

It seems natural to extend the above approach to elliptical galaxies and in particular to cluster dominant galaxies. Recent observations with the Hubble Space Telescope and groundbased telescopes have confirmed previous suggestions that 1) powerful low-redshift $(z<0.5)$ AGN are found in luminous and preferentially early-type galaxies (Bahcall et al. 1997; Hooper et al. 1997; Boyce et al. 1997; McLure et al. 1999; Schade et al. 2000) and 2) they are generally located in cluster environments (McLure \& Dunlop 2001). Since cluster cores typically have sizes $r_{\mathrm{c}} \sim 100 \mathrm{kpc}$, we could hope to detect radiation emitted by the nucleus (plus possibly by a jet) of a cluster dominant galaxy until a few times $r_{\mathrm{c}} / c \sim 10^{6}$ years ago and scattered by the hot intracluster gas toward us. Up to $\sim 1 \%$ of the radiation of the central source can be Thomson scattered by free electrons within the gas.

Previous theoretical efforts in this direction have focused on the prospects for detecting scattered (strongly polarized) AGN radiation at radio and optical wavelengths (Sunyaev 1982; Gilfanov et al. 1987a; Sholomitskii \& Yaskovich 1980; Wise \& Sarazin 1990, 1992; Sarazin \& Wise 1993; Murphy \& Chernoff 1993). However, it would be particularly interesting to get information on the history of the central source from observations of X-ray scattered radiation, since the bulk of the AGN X-ray emission is believed to originate within a few gravitational radii of the central black hole.

One feasible way to detect the scattered X-rays against the thermal emission of the hot gas would be to perform observations at $E \gg k T$, where $E$ is the photon energy and $k T \sim 1-10 \mathrm{keV}$ is the gas temperature. Indeed, the AGN spectrum is a power-law, $F_{\mathrm{AGN}} \propto E^{-\alpha}$ with $\alpha \sim 1$, while the plasma bremsstrahlung spectrum is exponentially declining, $F_{\text {gas }} \propto \exp (-E / k T)$, at $E \gg k T$. Unfortunately, the angular resolution and sensitivity of present-day hard X-ray telescopes are not sufficient for mapping clusters of galaxies and detecting scattered AGN radiation. However, the situation may improve dramatically with the advent of grazing incidence X-ray telescopes sensitive up to $\sim 40 \mathrm{keV}$, such as those under consideration for the projected Constellation- $\mathrm{X}^{1}$ and $\mathrm{XEUS}^{2}$ missions. Furthermore, given the huge collecting area (several square meters) of these planned telescopes in the standard X-ray band, it should become possible to search for scattered X-rays from distant quasars (at redshifts $z \gtrsim 1$ ). Indeed, since the radiation from a quasar gets redshifted on its way to us, a significant gain in the ratio $F_{\mathrm{AGN}} / F_{\text {gas }} \propto[E(1+z)]^{-\alpha} \exp [E(1+z) / k T]$ could be achieved already at $E \sim 10 \mathrm{keV}$. Such observations would permit one to constrain the lifetime of quasars and the parameters of the hot gas around them. This possibility is one of the issues discussed in the present paper (particularly in Sect. 3.2).

\footnotetext{
${ }^{1}$ http://constellation.gsfc.nasa.gov/docs/main.html

2 http://astro.esa.int/SA-general/Projects/XEUS/ main/xeus_main.html
}

On the observational side, evidence has been accumulated over recent years that AGN are often surrounded by gas atmospheres typical of clusters of galaxies. Early ROSAT observations indicated the presence of luminous extended X-ray emission around several powerful quasars with redshifts out to $z=0.73$ (Crawford et al. 1999; Hardcastle \& Worrall 1999). More recently, the Chandra observatory clearly resolved extended hot gas around several relatively nearby AGN, including 3C295 ( $z=0.461$, Harris et al. 2000), 3C220.1 ( $z=0.62$, Worral et al. 2001), 3C123 $(z=0.2177$, Hardcastle et al. 2001), Cyg A $(z=0.0562$, Smith et al. 2002), and H1821+643 $(z=0.297$, Fang et al. 2002).

Chandra has also detected luminous extended (to radii $\sim 100 \mathrm{kpc}$ ) X-ray emission surrounding two high-redshift powerful radio galaxies 3C294 at $z=1.786$ (Fabian et al. 2001) and PKS 1138-262 at $z=2.156$ (Carilly et al. 2002). Assuming for these objects that the emission of the central source is isotropic and its X-ray luminosity has been the same in the past as it is now ( $\sim$ a few $10^{45} \mathrm{erg} \mathrm{s}^{-1}$ ), we are able to estimate (see Sect. 3.2) that the gas surface brightness should be dominated by scattered power-law radiation from the AGN already at $E \gtrsim 10 \mathrm{keV}$.

In this paper we also discuss another possibility of detecting scattered AGN X-ray radiation. We show (in Sects. 2 and 3.3) that at least for nearby AGN located in cluster dominant galaxies or in isolated giant elliptical galaxies, one can gain significantly by measuring the scattered surface brightness in resonance X-ray lines rather than in the continuum. Such observations would ideally require high $(\sim \mathrm{a}$ few eV) spectral resolution in order to separate the resonance lines from other lines. The resolution needed together with high sensitivity can be achieved with future high-energy astrophysics observatories such as Astro-E2 ${ }^{3}$, Constellation-X and XEUS. As an example, we estimate (in Sect. 4) the level of constraints it should be possible to derive on the past X-ray luminosity of the nearby M 87 and Cyg A active galaxies. Yet better constraints could be obtained by measuring the polarization of the scattered Xray resonance line radiation (see Sect. 5; Sazonov et al. 2002).

\section{Advantage of scattered X-ray lines over the scattered continuum}

Consider an AGN surrounded by hot $\left(T \gtrsim 10^{7} \mathrm{~K}\right)$ gas. The following emission components will contribute to the X-ray surface brightness of the gas:

- First, there is X-ray emission (line plus continuum) of the hot plasma in which collisional ionization and excitation dominate. However, a bright AGN may additionally photoionize the plasma. In our calculations below we fully neglect this effect, although it can be important when the AGN luminosity is very high (quasar-like).

- Second, there is radiation emitted by the AGN and Thomson scattered by free electrons in the gas. This scattered radiation contributes to the surface brightness in the spectral continuum.

\footnotetext{
${ }^{3}$ http://www.astro.isas.ac.jp/astroe/index-e.html
} 
- Finally, AGN photons with energies falling within the cores of X-ray lines of ions of heavy elements can resonantly scatter on these ions, thus contributing to the surface brightness in the lines.

Consider an optically thin volume of gas with electron temperature $T$ and number density $n_{\mathrm{e}}$, exposed to an external spectral flux $F_{E}(E)$ (measured in units of erg $\mathrm{cm}^{-2} \mathrm{~s}^{-1} \mathrm{keV}^{-1}$ ).

The plasma continuum spectral emissivity due to bremsstrahlung is given by (e.g. Zombeck 1990)

$$
\begin{array}{r}
\epsilon_{E, \text { cont }}(E)=2.3 \times 10^{-20} T^{-1 / 2} \exp (-E / k T) n_{\mathrm{e}}^{2} g_{\mathrm{B}}(T, E) \\
\left(\operatorname{erg~cm}{ }^{-3} \mathrm{~s}^{-1} \mathrm{keV}^{-1}\right),
\end{array}
$$

where $g_{\mathrm{B}}$ is the corresponding Gaunt factor.

The energy-integrated emissivity in a resonance line by ions of type $z$ due to electron collisional excitation of an electron from the ground level $i$ to an excited level $k$ is given by (e.g. Zombeck 1990)

$$
\begin{aligned}
\epsilon_{\text {line }}= & 2.7 \times 10^{-15} T^{-1 / 2} \exp \left(-E_{i k} / k T\right) \\
& \times n_{\mathrm{e}} n_{z}(T) f_{i k} g_{i k}(T)\left(\mathrm{erg} \mathrm{cm}^{-3} \mathrm{~s}^{-1}\right),
\end{aligned}
$$

where $n_{z}$ is the ion number density, which depends on the gas temperature. The remaining quantities appearing in Eq. (2), namely $E_{i k}, f_{i k}$ and $g_{i k}$, characterize the line itself, denoting its energy, oscillator strength and excitation Gaunt factor, respectively.

The rate (per unit volume) of Thomson scattering of the external radiation, integrated over the scattering angle $\theta$, is

$$
\begin{aligned}
\epsilon_{E, \mathrm{cont}}^{\text {scat }}(E) & =\frac{3}{8} \int_{-1}^{1} \mathrm{~d} \cos \theta\left(1+\cos ^{2} \theta\right) \epsilon_{E, \theta, \mathrm{cont}}^{\text {scat }}(E, \theta) \\
& =F_{E}(E) n_{\mathrm{e}} \sigma_{\mathrm{T}}\left(\mathrm{erg} \mathrm{cm}^{-3} \mathrm{~s}^{-1} \mathrm{keV}^{-1}\right)
\end{aligned}
$$

where $\sigma_{\mathrm{T}}=6.65 \times 10^{-25}$ is the Thomson scattering cross section. Note that we ignore throughout the Klein-Nishina correction to the scattering cross section as well as the change in the photon energy by scattering, which is justifiable if $E \ll m c^{2}$ and $k T \ll m c^{2}$, where $m c^{2}=511 \mathrm{keV}$ is the electron rest energy.

The energy-integrated rate of resonant scattering (integrated over the scattering angle) of the external radiation in the line $i \rightarrow k$ by ions $z$ is

$\epsilon_{\text {line }}^{\text {scat }}=4.1 \times 10^{-18} \pi F_{E}\left(E_{i k}\right) n_{z}(T) r_{\mathrm{e}} c f_{i k}\left(\operatorname{erg~cm}^{-3} \mathrm{~s}^{-1}\right)$,

where $r_{\mathrm{e}}=2.82 \times 10^{-13} \mathrm{~cm}$ is the classical electron radius, $c$ is the speed of light, and the numerical coefficient accounts for the unit transition from $\mathrm{Hz}$ to $\mathrm{keV}$.

From Eqs. (1) and (3) we can find the ratio of the Thomson scattering rate to the bremsstrahlung emissivity:

$\frac{\epsilon_{E, \text { cont }}^{\text {scat }}}{\epsilon_{E \text {, cont }}}(E)=2.9 \times 10^{-5} F_{E}(E) T^{1 / 2} \exp (E / k T) n_{\mathrm{e}}^{-1} g_{\mathrm{B}}^{-1}(T, E)$.

The ratio of the resonant scattering rate to the emissivity in the line $i \rightarrow k$ is given by a similar expression, which follows from Eqs. (2) and (4),

$\frac{\epsilon_{\text {line }}^{\text {scat }}}{\epsilon_{\text {line }}}=4.1 \times 10^{-5} F_{E}\left(E_{i k}\right) T^{1 / 2} \exp \left(E_{i k} / k T\right) n_{\mathrm{e}}^{-1} g_{i k}^{-1}(T)$.

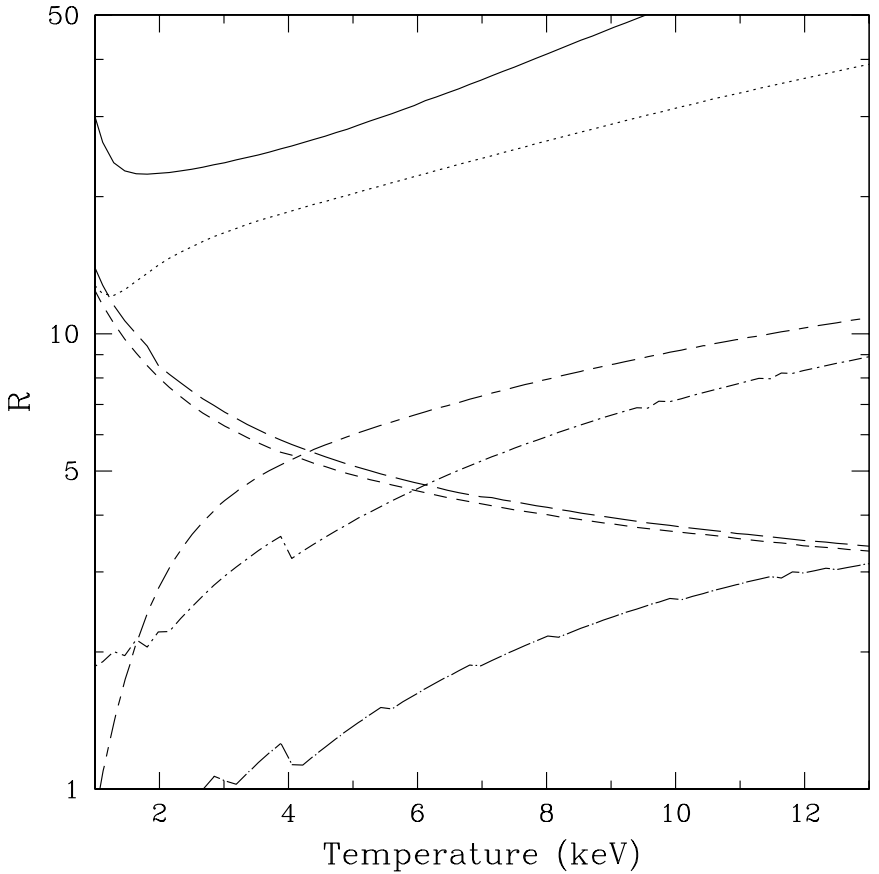

Fig. 1. Ratio $R$ defined by Eq. (7) as a function of plasma temperature for various resonance X-ray lines: Fe XXIII L-shell line at $1.13 \mathrm{keV}$ (solid), Fe XXIV L-shell line at $1.17 \mathrm{keV}$ (dotted), Si XIII K $\alpha$ line at $1.86 \mathrm{keV}$ (short-dashed), S XV K $\alpha$ line at $2.46 \mathrm{keV}$ (long-dashed), $\mathrm{Fe} \mathrm{XXV} \mathrm{K} \alpha$ line at $6.70 \mathrm{keV}$ (dot-short-dashed), Fe XXVI Ly $\alpha$ line at $6.97 \mathrm{keV}$ (dot-long-dashed), and Fe XXV K $\beta$ line at $7.88 \mathrm{keV}$ (shortdash-long-dashed).

We can now compare the ratios (6) and (5) for $E=E_{i k}$ :

$R \equiv \frac{\epsilon_{\text {line }}^{\text {scat }}}{\epsilon_{\text {line }}} / \frac{\epsilon_{E, \text { cont }}^{\text {scat }}}{\epsilon_{E, \text { cont }}}=1.4 \frac{g_{\mathrm{B}}\left(T, E_{i k}\right)}{g_{i k}(T)}$.

In the low-temperature limit $\left(k T \ll E_{i k}\right)$, the excitation Gaunt factor $g_{i k}$ ranges between 0.1 and 0.25 for most interesting lines; it slowly increases with temperature and reaches $0.2-0.3$ at $k T \sim E_{i k}$ (e.g. Mewe et al. 1985). The bremsstrahlung Gaunt factor also is a weak function of temperature: $g_{\mathrm{B}}=(k T / E)^{0.4}$ when $E \sim k T$. Summarizing these facts, we may simply write

$R \sim 6$ for $k T \sim E_{i k}$.

This approximate relation is accurate to within a factor of 3 for all resonance X-ray lines of interest to us. Figure 1 shows, for several resonance lines, the dependence $R(T)$ computed using the MEKAL plasma emission code in combination with other atomic data sources (see Sect. 4 for details). In these computations the contribution to $\epsilon_{\text {line }}$ of unresolved (defined as those with energies within one Doppler width of the resonance energy) satellite lines was taken into account. This effectively leads to a smaller value of $R$ compared to the definition (7). However, this reduction is only significant for the $6.70 \mathrm{keV}$ and $6.97 \mathrm{keV} \mathrm{K} \alpha$ lines or iron when $k T \ll E_{i k}$. We should note that the large values $\left(R \sim 20\right.$ at $\left.k T \sim E_{i k}\right)$ obtained for the iron Lshell lines at $1.13 \mathrm{keV}$ and $1.17 \mathrm{keV}$ may be partly the result of the different values for the oscillation strengths being used in the MEKAL code with which we compute $\epsilon_{\text {line }}$ and in the list 
of resonance lines of Verner et al. (1996) which we use to compute $\epsilon_{\text {line }}^{\text {scat }}$. We do not attempt in this paper to correct for this and possibly other inconsistencies caused by the simultaneous use of several sources of atomic data. However, we estimate that some of our computational results obtained below (in Sect. 4) may contain a relative systematic error of $\lesssim 2$.

We point out that $R$ is typically several times smaller for intercombination lines than for resonance lines and $R \approx 0$ for forbidden lines. For this reason we consider only resonance lines throughout.

We have thus found that for a given resonance line with energy $E_{i k}$, the relative contribution of scattered external radiation to the volume X-ray emissivity of hot gas is typically larger by a significant factor of the order of 3-10 than for the continuum at $E_{i k}$. We also know that for typical cluster temperatures $k T \sim$ a few $\mathrm{keV}$ the combined surface brightness of the intracluster gas in the resonance X-ray lines with $E_{i k} \sim k T$ is comparable to that in the continuum. We therefore arrive at the conclusion that resonance X-ray lines present significant advantage over the continuum for searches of scattered AGN radiation in clusters of galaxies and elliptical galaxies performed at $E \sim k T$.

After we have introduced the $R$ factor in Eq. (7) and shown that this factor is only weakly dependent on the resonance line and on gas temperature, we can continue our treatment in parallel for continuum and line emission.

\section{AGN in the center of a beta-cluster}

We shall now consider the following model (see Fig. 2). A supermassive black hole is located in the center of a cluster of galaxies containing hot gas with a beta-law radial density profile (Cavaliere \& Fusco-Femiano 1976):

$n_{\mathrm{e}}=n_{0}\left(1+r^{2} / r_{\mathrm{c}}^{2}\right)^{-3 \beta / 2}$,

where $n_{0}$ is the central electron number density and $r_{\mathrm{c}}$ is the core-radius. The gas temperature $T$ and element abundances are constant over the cluster.

At a moment $t_{\mathrm{on}}=-\Delta$ (as measured by an earth bound observer) the central source switches on (i.e. becomes an AGN) and at a later moment $t_{\mathrm{off}}=0$ it switches off. The AGN emits isotropically $\mathrm{X}$-ray radiation having a power-law spectrum of photon index $\gamma$, at a constant luminosity $L_{X}$ in the energy band $\left[E_{1}, E_{2}\right]$ :

$L_{X}=A \int_{E_{1}}^{E_{2}} E^{-\gamma} E \mathrm{~d} E=A \frac{E_{2}^{2-\gamma}-E_{1}^{2-\gamma}}{2-\gamma}$.

The spectral flux at a given distance $r$ from the AGN is therefore

$F_{E}=\frac{(2-\gamma) L_{X}}{4 \pi\left(E_{2}^{2-\gamma}-E_{1}^{2-\gamma}\right) r^{2}} E^{1-\gamma}$.

Our goal is to find the surface brightness profile of the cluster, including the contribution of scattered AGN radiation, at a given moment of time $t>t_{\mathrm{on}}$.

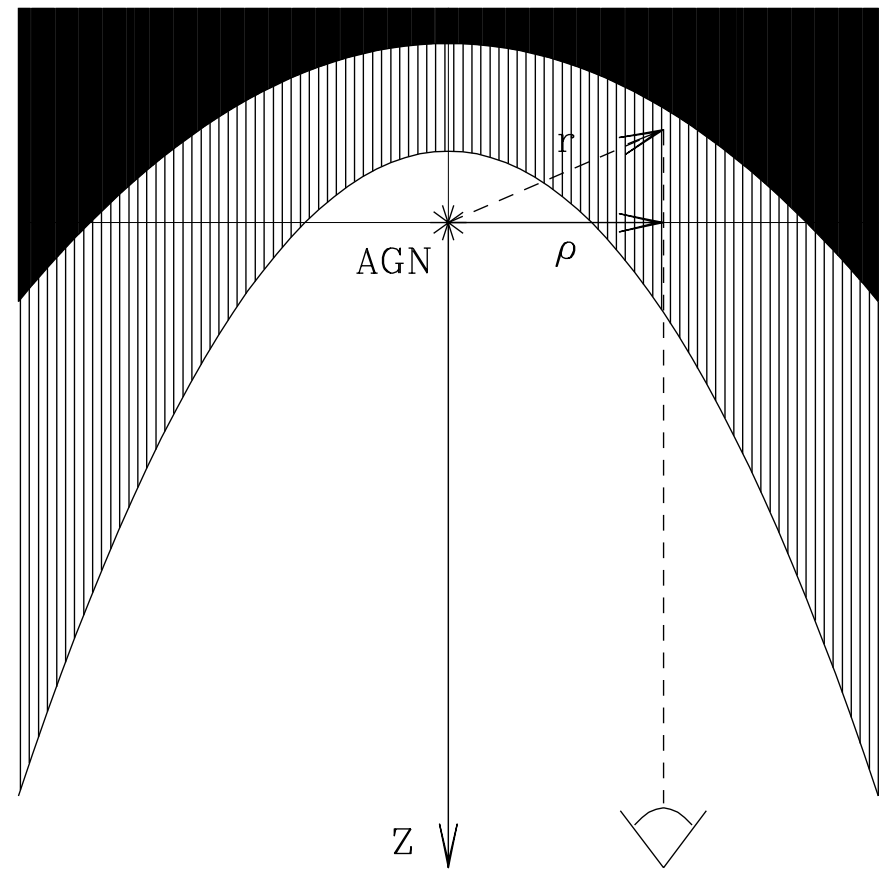

Fig. 2. Sketch of the model. The points with equal travel time from the AGN to the observer lie on a paraboloid. In the switch-off case, the thermal plasma located in the grey and black regions is scattering the radiation emitted by the AGN. In the case of a flare of the AGN, only the grey area is filled by photons and contributing to the scattered emission. A possible path for a photon scattered into the line of sight of the observer is marked by a dashed line. The system is rotationally symmetric about the $z$-axis.

\subsection{Approximation of isotropic scattering}

We shall use throughout this paper the approximation of isotropic scattering. This simplifies the treatment but requires explanation. We know that in reality Thomson scattering occurs according to the Rayleigh phase function $p(\cos \theta)=$ $3\left(1+\cos ^{2} \theta\right) / 8$. In the case of resonant scattering, the phase function depends on the line and generally can be represented as a weighted sum of the Rayleigh phase function and the isotropic function $p(\cos \theta)=1 / 2$ (see e.g. Chandrasekhar 1950). However, for our given geometry of the problem the resulting brightness profiles depend only weakly (typically by less than $10 \%$ ) on the phase function. For this reason, the approximation of isotropic scattering is quite reasonable.

\subsection{Continuum radiation}

We shall first consider the X-ray spectral continuum, for which the relevant scattering mechanism is Thomson scattering. Since the Thomson optical depths of clusters of galaxies and elliptical galaxies are small, $\tau_{\mathrm{T}} \lesssim 0.01$, we can use the single-scattering approximation and make some analytic estimates.

The continuum (bremsstrahlung) surface brightness of a beta-cluster is described, as a function of projected distance $\rho$ from the nucleus, by the well-known formula, which can be obtained by integrating the plasma emission along a given line 
of sight (i.e. along the coordinate $z$ - see Fig. 2) using Eqs. (1) and (9):

$$
\begin{aligned}
& B_{E, \text { cont }} \equiv \int_{-\infty}^{\infty} \frac{\epsilon_{E, \text { cont }}(r)}{4 \pi} \mathrm{d} z \\
& =2.9 \times 10^{-5} \frac{\Gamma(3 \beta-1 / 2)}{\Gamma(3 \beta)}\left(\frac{n_{0}}{0.01 \mathrm{~cm}^{-3}}\right)^{2} \frac{r_{\mathrm{c}}}{100 \mathrm{kpc}} \\
& \times\left(\frac{k T}{1 \mathrm{keV}}\right)^{-1 / 2}(E / k T)^{-0.4} \exp (-E / k T) \\
& \times\left(1+\rho^{2} / r_{\mathrm{c}}^{2}\right)^{-3 \beta+1 / 2} \\
& \left(\operatorname{erg~cm}{ }^{-2} \mathrm{~s}^{-1} \mathrm{sr}^{-1} \mathrm{keV}^{-1}\right) \text {, }
\end{aligned}
$$

where $r=\left(\rho^{2}+z^{2}\right)^{1 / 2}$ and we have adopted $g_{\mathrm{B}}(T, E)=$ $(E / k T)^{-0.4}$.

The surface brightness of the Thomson scattered AGN radiation is given by

$B_{E, \text { cont }}^{\text {scat }} \equiv \int_{z_{1}}^{z_{2}} \frac{\epsilon_{E, \text { cont }}^{\text {scat }}(r)}{4 \pi} \mathrm{d} z=\frac{\sigma_{\mathrm{T}}}{4 \pi} \int_{z_{1}}^{z_{2}} F_{E}(r) n_{\mathrm{e}}(r) \mathrm{d} z$,

where we have used Eq. (3) and our assumption of isotropic scattering.

The time-dependent integration limits $z_{1}(t, \rho)$ and $z_{2}(t, \rho)$ in Eq. (13) are determined by the loci of scattering sites giving a fixed time delay $\tilde{t}$. If the distance between the emitter and the observer is much larger than the characteristic size of the scattering cloud, which is true in our case, the constant-delay surface is a paraboloid with its focus at the position of the source (see e.g. Sunyaev \& Churazov 1998 and references therein to earlier work considering similar light-echo problems):

$z(\tilde{t}, \rho)=\frac{1}{2}\left(\frac{\rho^{2}}{c \tilde{t}}-c \tilde{t}\right)$.

Therefore,

$z_{1}(t, \rho)=\frac{1}{2}\left[\frac{\rho^{2}}{c(t+\Delta)}-c(t+\Delta)\right]$,

$z_{2}(t, \rho)= \begin{cases}\left(\rho^{2} / c t-c t\right) / 2 & \text { if } 0<t \\ \infty & \text { if }-\Delta<t<0 .\end{cases}$

The integration limits in Eq. (13) depend on the outburst duration $\Delta$. We shall first consider two limiting cases for which analytic treatment is possible.

\subsubsection{Stationary case}

Let the AGN be a persistent source. The limits of the integral $\int_{z_{1}}^{z_{2}} \epsilon_{E, \text { cont }}^{\text {scat }} \mathrm{d} z$ are then $z_{1}(t, \rho)=-\infty$ and $z_{2}(t, \rho)=\infty$, and it is possible, using Eq. (9) for $n_{\mathrm{e}}(r)$ and Eq. (11) for $F_{E}$, to express the integral through hypergeometric functions. In the particular interesting case of $\beta=2 / 3$, the scattered surface brightness is

$$
\begin{aligned}
& B_{E, \text { cont }}^{\text {scat }}(\beta=2 / 3)=6.0 \times 10^{-5} \frac{L_{X}}{L_{\mathrm{Edd}}} \frac{M_{\mathrm{BH}}}{10^{9} M_{\odot}} \frac{n_{0}}{0.01 \mathrm{~cm}^{-3}} \\
& \times \frac{100 \mathrm{kpc}}{r_{\mathrm{c}}} \frac{2-\gamma}{10^{2-\gamma}-1}\left[r_{\mathrm{c}} / \rho-\left(1+\rho^{2} / r_{\mathrm{c}}^{2}\right)^{-1 / 2}\right] E^{1-\gamma} \\
& \left(\operatorname{erg~cm}{ }^{-2} \mathrm{~s}^{-1} \mathrm{sr}^{-1} \mathrm{keV}^{-1}\right) \text {, }
\end{aligned}
$$

where $M_{\mathrm{BH}}$ is the mass of the central black hole, $L_{\mathrm{Edd}}=1.4 \times$ $10^{38} M_{\mathrm{BH}} / M_{\odot}$ is the corresponding Eddington luminosity, $L_{X}$ is the AGN luminosity in the energy range $1-10 \mathrm{keV}$, and $E$ is measured in $\mathrm{keV}$.

The ratio of the scattered surface brightness to the intrinsic surface brightness of the gas in the continuum is

$$
\begin{aligned}
\frac{B_{E, \mathrm{cont}}^{\text {scat }}}{B_{E, \mathrm{cont}}}(\beta=2 / 3)= & 2.3 \frac{L_{X}}{L_{\mathrm{Edd}}} \frac{M_{\mathrm{BH}}}{10^{9} M_{\odot}} \frac{0.01 \mathrm{~cm}^{-3}}{n_{0}} \\
& \times\left(\frac{100 \mathrm{kpc}}{r_{\mathrm{c}}}\right)^{2}\left(\frac{k T}{1 \mathrm{keV}}\right)^{0.1} \frac{2-\gamma}{10^{2-\gamma}-1} \\
& \times\left(1+\rho^{2} / r_{\mathrm{c}}^{2}\right)^{3 / 2}\left[r_{\mathrm{c}} / \rho-\left(1+\rho^{2} / r_{\mathrm{c}}^{2}\right)^{-1 / 2}\right] \\
& \times E^{1.4-\gamma} \exp (E / k T) .
\end{aligned}
$$

We can also write down asymptotic expressions applicable in the limit of $\rho \gg r_{\mathrm{c}}$ for arbitrary values of $\beta$ :

$$
\begin{aligned}
& B_{E, \text { cont }}^{\text {scat }}(\rho \gg\left.r_{\mathrm{c}}\right)=3.2 \times 10^{-5} \frac{\Gamma(3 \beta / 2+1 / 2)}{\Gamma(3 \beta / 2+1)} \frac{L_{X}}{L_{\mathrm{Edd}}} \frac{M_{\mathrm{BH}}}{10^{9} M_{\odot}} \\
& \times \frac{n_{0}}{0.01 \mathrm{~cm}^{-3}} \frac{100 \mathrm{kpc}}{r_{\mathrm{c}}} \frac{2-\gamma}{10^{2-\gamma}-1}\left(\rho / r_{\mathrm{c}}\right)^{-3 \beta-1} E^{\gamma-1} \\
&\left(\mathrm{erg} \mathrm{cm}^{-2} \mathrm{~s}^{-1} \mathrm{sr}^{-1} \mathrm{keV}^{-1}\right) ;
\end{aligned}
$$

$$
\begin{aligned}
\frac{B_{E, \text { cont }}^{\text {scat }}}{B_{E, \text { cont }}}\left(\rho \gg r_{\mathrm{c}}\right) & =1.1 \frac{\Gamma(3 \beta / 2+1 / 2) \Gamma(3 \beta)}{\Gamma(3 \beta / 2+1) \Gamma(3 \beta-1 / 2)} \frac{L_{X}}{L_{\mathrm{Edd}}} \\
& \times \frac{M_{\mathrm{BH}}}{10^{9} M_{\odot}} \frac{0.01 \mathrm{~cm}^{-3}}{n_{0}}\left(\frac{100 \mathrm{kpc}}{r_{\mathrm{c}}}\right)^{2}\left(\frac{k T}{1 \mathrm{keV}}\right)^{0.1} \\
& \times \frac{2-\gamma}{10^{2-\gamma}-1}\left(\rho / r_{\mathrm{c}}\right)^{3 \beta-2} E^{1.4-\gamma} \exp (E / k T)
\end{aligned}
$$

It can also be shown that within the cluster core, $B_{E, \text { cont }}^{\text {scat }} \propto \rho^{-1}$ and $B_{E, \text { cont }}^{\text {scat }} / B_{E, \text { cont }} \propto \rho^{-1}$ for any $\beta$. This can be directly verified for $\beta=2 / 3$ using Eq. (18).

Figure $3 \mathrm{a}$ demonstrates the dependence of the surface brightness profile of a cluster with a central AGN on the $\beta$ parameter. The scattered surface brightness profiles were derived by numerical integration of Eq. (13). Figure 4 shows the corresponding plots for the ratio of the scattered surface brightness to the intrinsic surface brightness of the intracluster gas. We see both from Fig. 4 and Eq. (20) that outside the core region (at $\rho \gtrsim r_{\mathrm{c}}$ ), the relative contribution of scattered AGN radiation to the surface brightness increases with $\rho$ when $\beta>2 / 3$.

The $\beta=1 / 3$ case presented in Figs. 3a and 4 corresponds to cluster cooling flows. Typically, gas density grows $\propto r^{-1}$ toward the center of the cooling flow, which roughly corresponds to a beta-model with a small $r_{\mathrm{c}}$, large $n_{0}$ and $\beta \approx 1 / 3$. We then find from Eqs. (12) and (19) that $B_{E \text {,cont }} \propto \rho^{-1}$ and $B_{E, \text { cont }}^{\text {scat }} \propto \rho^{-2}$ (assuming a constant temperature) for cooling flows. Interestingly, the surface brightness profile of scattered AGN radiation within the core of a beta-cluster (see above) is similar $\left(\propto \rho^{-1}\right)$ to the intrinsic brightness profile of an (isothermal) cooling flow. Of course, a cluster core dominated by scattered emission from a central powerful quasar will have a very different spectrum (power law) than a cooling flow.

The energy dependent factor $E^{1.4-\gamma} \exp (E / k T)$ appearing in Eqs. (18) and (20) has a minimum (for $\gamma>1.4$ ) at 


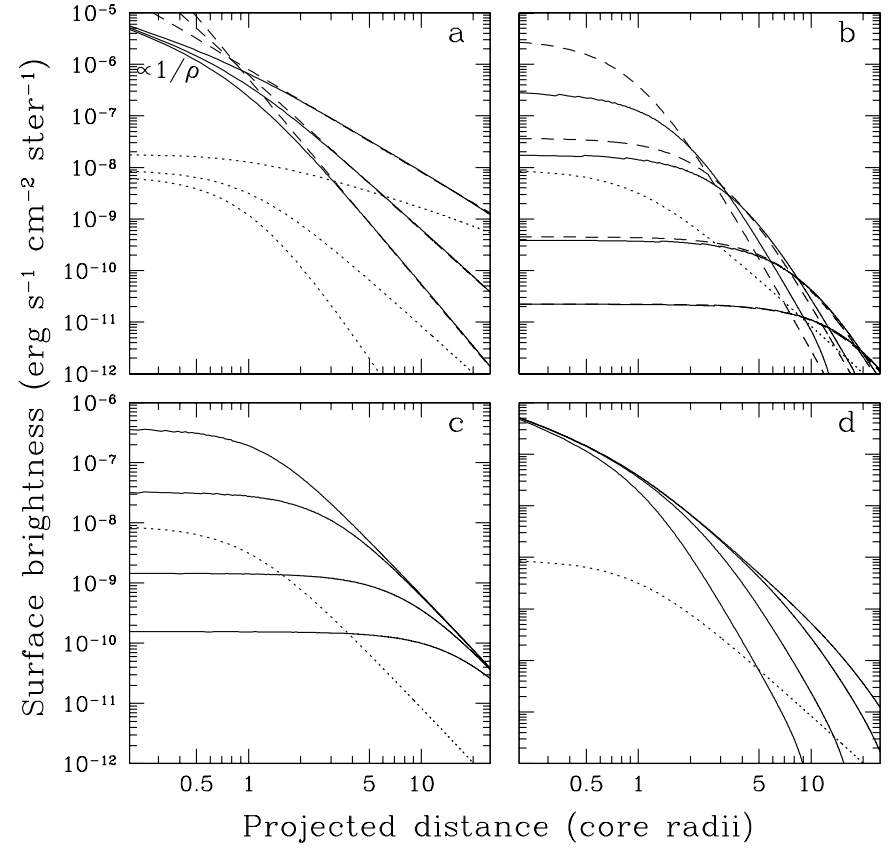

Fig. 3. a) Surface brightness profiles of scattered AGN radiation (solid lines) and plasma thermal emission (dotted lines) in the X-ray continuum at $E=20 \mathrm{keV}$, for a model in which a central $10^{9} M_{\odot}$ black hole is a persistent X-ray source with $L_{X}=L_{\mathrm{Edd}}$ and $\gamma=2$. The host cluster has the following parameters: $r_{\mathrm{c}}=100 \mathrm{kpc}, n_{0}=0.01 \mathrm{~cm}^{-3}$, $k T=3 \mathrm{keV}$, and (from top to bottom) $\beta=1 / 3,2 / 3$ and 1 , and is local $(z \ll 1)$. The dashed lines are $\rho \gg r_{\mathrm{c}}$ asymptotes given by Eq. (19), applicable at $\rho \gtrsim 2 r_{\mathrm{c}}$. In the opposite limit of $\rho \ll 1$ the scattered surface brightness is proportional to $\rho^{-1}$ and is independent of $\beta$, as indicated in the upper left corner of the figure. b) Surface brightness profiles of scattered AGN radiation (solid lines) at $E=20 \mathrm{keV}$, measured at different times $\left(t=r_{\mathrm{c}} / c, 3 r_{\mathrm{c}} / c, 9 r_{\mathrm{c}} / c\right.$ and $19 r_{\mathrm{c}} / c-$ from top to bottom) after the end of a short $\left(\Delta=r_{\mathrm{c}} / c\right)$ flare with $L_{X}=L_{\mathrm{Edd}}$. For the same values of the parameters as in a) and $\beta=2 / 3$. The dashed lines are $\rho, c t \gg r_{\mathrm{c}}$ asymptotes given by Eq. (22). The dotted line is the surface brightness profile of plasma thermal emission. c) Same as b), but for the case where the central source was on $\left(L_{X}=L_{\mathrm{Edd}}\right)$ until $t_{\text {off }}=0$ (profiles order from top to bottom). d) Same as c), but for the case where the central AGN switched on at $t=0$ (profiles order from bottom to top).

$E=(\gamma-1.4) k T$ and increases exponentially when $E \gg k T$. The origin of this dependence is obvious: we are comparing the slowly declining (power-law) spectrum of the AGN emission with the exponentially decaying efficiency of collisional radiative mechanisms operating in the intracluster gas. It is clear that for given AGN luminosity and parameters of the gas, a significant gain (in terms of the relative contribution of scattered AGN radiation to the surface brightness) can be achieved by performing observations at $E \gg k T$, like in our examples in Figs. 3 and 4. A cautionary note is necessary here. If one wants to constrain the past luminosity of an AGN by mapping its host elliptical galaxy in hard X-rays, a restriction will appear due to a significant contribution to the measured surface brightness from the (unresolved) X-ray binaries of the galaxy. We estimate that in practice it should be very difficult to obtain limits better than $L_{X} \sim 10^{-3} L_{\text {Edd }}$. Of course, this complication is irrelevant for surface brightness measurements carried out

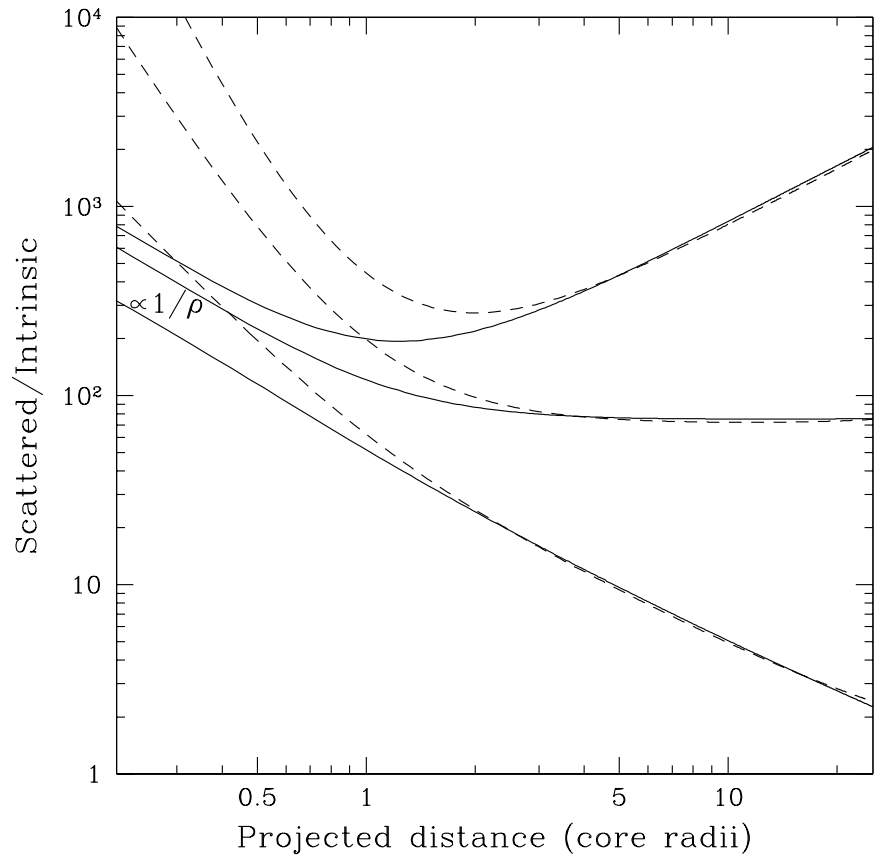

Fig. 4. Radial profiles of the ratio of the surface brightness of scattered AGN radiation to the intrinsic brightness of the beta-cluster gas, for the same model as in Fig. 3a. The solid lines correspond (from bottom to top) to $\beta=1 / 3,2 / 3$ and 1 . The dashed lines are the corresponding $\rho \gg r_{\mathrm{c}}$ asymptotes given by Eq. (20).

over regions of a galaxy cluster that are almost devoid of point $\mathrm{X}$-ray sources, say at distances $\sim 100 \mathrm{kpc}$ from the nucleus of a central dominant galaxy.

\subsubsection{The case of a high-redshift AGN}

Furthermore, if a powerful quasar is observed at high redshift $z$, the relevant factor will be $[E(1+z)]^{1.4-\gamma} \exp [E(1+z) / k T]$. Consider for example the radio galaxy 3C294 located at $z=$ 1.786 , for which a surrounding intracluster medium has recently been revealed with Chandra (Fabian et al. 2001). Since the total number of counts registered from the source in the 20 ksec Chandra observation was small, 100 , of which only $\sim 30$ are from the central AGN, it is only possible to make rough estimates in this case. Fabian et al. find that the gas temperature is $\sim 5 \mathrm{keV}$, the $2-10 \mathrm{keV}$ rest-frame luminosity of the hot gas is $\sim 2.5 \times 10^{44} \mathrm{erg} \mathrm{s}^{-1}$, the corresponding (isotropic) luminosity of the AGN is $\sim 1.1 \times 10^{45} \mathrm{erg} \mathrm{s}^{-1}$, the photon index for the AGN emission $\gamma \sim 2$ and the radial Thomson depth of the cluster is $\sim 0.004$.

We can then estimate that the ratio of the flux of scattered AGN radiation to that of thermal bremsstrahlung emission from the gas is only $\sim 1 \%$ at $E \lesssim 2 \mathrm{keV}, \sim 3 \%$ at $5 \mathrm{keV}, \sim 30 \%$ at $10 \mathrm{keV}, \sim 1$ at $12 \mathrm{keV}$ and $\sim 4$ at $15 \mathrm{keV}$. These estimates assume that the central source is isotropic and its luminosity has been the same in the recent past (over $\sim$ a few $10^{5}$ years), and are made for the total flux from the whole observed $\mathrm{X}$-ray halo (of $\sim 15 \mathrm{arcsec}$, or equivalently $\sim 100 \mathrm{kpc}$ radius). We thus see that it is desirable to go to energies above $10 \mathrm{keV}$ 
in searching for scattered AGN radiation around the 3C294 galaxy. However, photon statistics then becomes a limiting factor. We can roughly estimate that the XEUS observatory with its effective area of $\sim 2 \mathrm{~m}^{2}$ at $10 \mathrm{keV}$ and angular resolution of a few arcsec should be able to detect only a total of $\sim 10$ scattered AGN photons above $10 \mathrm{keV}$ in a $100 \mathrm{ks}$ observation of $3 \mathrm{C} 294$. This, combined with much more accurate measurements of the surface brightness of the intracluster gas in the standard X-ray band, could be only about enough to put constraints on the past history of the 3C294 central X-ray luminosity.

Another similar example is the radio galaxy PKS 1138-262 at $z=2.2$, from which Chandra also has observed luminous extended X-ray emission (Carilly et al. 2002). In this case, the gas X-ray luminosity is similar to that of the 3C294 cluster (unfortunately, the gas temperature is unconstrained), but the inferred 2-10 keV (rest-frame) luminosity of the central source is four times higher, $\sim 4 \times 10^{45} \mathrm{erg} \mathrm{s}^{-1}$. Moreover, in this case the gas emission is likely dominated by scattered AGN radiation already at $E \gtrsim 5 \mathrm{keV}$ (due to the higher redshift and luminosity), so that a few hundred scattered AGN photons could be detected above $5 \mathrm{keV}$ in a $100 \mathrm{ks}$ XEUS-2 observation of PKS 1138-262 (taking into account the larger collecting area of the telescope at lower energies).

We note that photon statistics should be less of a problem for more nearby AGN and quasars such as 3C 273 located at $z=$ 0.158 . In fact, it is possible, given the huge X-ray luminosity of 3C $273, \sim 2 \times 10^{46} \mathrm{erg} \mathrm{s}^{-1}$ (e.g. Yaqoob \& Serlemitsos 1990), that a hot intracluster medium surrounding it, if any, will be first observed in scattered rather its own X-ray emission.

\subsubsection{Short AGN flare}

Suppose now that an AGN experienced an outburst in the past, between $t_{\mathrm{on}}=-\Delta$ and $t_{\mathrm{off}}=0$. The "short" here means that the line-of-sight depth of the illuminated (see Fig. 2) zone of the cluster must be much smaller than the characteristic size at a given projection radius. Therefore, one of the following conditions must be fulfilled: $\Delta \ll r_{\mathrm{c}} / c$ if $\rho \lesssim r_{\mathrm{c}}$ or $\Delta \ll \rho / c$ if $\rho \gtrsim r_{\mathrm{c}}$. Then, as follows from Eqs. (15) and (16),

$\delta z(t, \rho) \equiv z_{2}-z_{1} \approx \frac{c \Delta}{2}\left(1+\rho^{2} / c^{2} t^{2}\right)$,

and $\int_{z_{1}}^{z_{2}} \epsilon_{E, \text { cont }}^{\text {scat }}(\rho, z) \mathrm{d} z \approx \epsilon_{E \text {,cont }}^{\text {scat }}\left(\rho, z_{2}\right) \delta z(\rho)$. We then find in the limit of $\rho, c t \gg r_{\mathrm{c}}$

$$
\begin{aligned}
B_{E, \text { cont }}^{\text {scat }}= & 3.2 \times 10^{-5} \times 4^{3 \beta / 2} \frac{L_{X}}{L_{\mathrm{Edd}}} \frac{M_{\mathrm{BH}}}{10^{9} M_{\odot}} \\
& \times \frac{n_{0}}{0.01 \mathrm{~cm}^{-3}} \frac{100 \mathrm{kpc}}{r_{\mathrm{c}}} \frac{2-\gamma}{10^{2-\gamma}-1} \\
& \times(\Delta / t)\left(r_{\mathrm{c}} / c t\right)^{3 \beta+1}\left(1+\rho^{2} / c^{2} t^{2}\right)^{-3 \beta-1} E^{1-\gamma} \\
& \left(\mathrm{erg} \mathrm{cm}^{-2} \mathrm{~s}^{-1} \mathrm{sr}^{-1} \mathrm{keV}^{-1}\right)
\end{aligned}
$$

$$
\begin{aligned}
\frac{B_{E, \text { cont }}^{\text {scat }}}{B_{E, \text { cont }}}= & 1.2 \times 4^{3 \beta / 2} \frac{\Gamma(3 \beta)}{\Gamma(3 \beta-1 / 2)} \frac{L_{X}}{L_{\mathrm{Edd}}} \frac{M_{\mathrm{BH}}}{10^{9} M_{\odot}} \\
& \times \frac{0.01 \mathrm{~cm}^{-3}}{n_{0}}\left(\frac{100 \mathrm{kpc}}{r_{\mathrm{c}}}\right)^{2}\left(\frac{k T}{1 \mathrm{keV}}\right)^{0.1} \frac{2-\gamma}{10^{2-\gamma}-1} \\
& \times(\Delta / t)\left(c t / r_{\mathrm{c}}\right)^{3 \beta-2}(\rho / c t)^{6 \beta-1}\left(1+\rho^{2} / c^{2} t^{2}\right)^{-3 \beta-1} \\
& \times E^{1.4-\gamma} \exp (E / k T) .
\end{aligned}
$$

Comparing Eqs. (23) and (20), we see that for a given luminosity $L_{X}$ the contribution of scattered AGN radiation to the surface brightness at $\rho \sim c t$ is smaller by a factor of $\Delta / t$ for the flare compared to the stationary case. This happens because in the former case the scattered radiation comes from a gas layer of depth $\delta z \sim c \Delta$ along the line of sight, whereas $\delta z \sim \rho \sim c t$ in the latter case.

Figure $3 \mathrm{~b}$ shows a sequence of scattered surface brightness profiles that would be measured at different times after a short AGN outburst. We see that distant regions of the cluster reveal no scattered light early on after the flare but become progressively brighter as time goes by and photons of the flare propagate through the gas. The ratio $B_{E \text { cont }}^{\text {scat }} / B_{E \text {,cont }}$ has a maximum at $\rho_{\max } \sim c t$, as indicated by Eq. (23). For example, $\rho_{\max }=r_{\mathrm{c}}$ when $\beta=2 / 3$. Therefore, the zone of largest contribution of scattered AGN radiation propagates outward at an apparent velocity equal to the speed of light. It is important for observations that this zone is broad $-\delta \rho_{\mathrm{eff}} \sim \rho_{\mathrm{max}} \sim c t$.

We also notice that the amplitude of the effect is proportional to the product $L_{X} \Delta$, i.e. to the total energy emitted by the AGN during the flare. Thus, for example, a $10^{5}$-year outburst at $L_{X}=0.01 L_{\text {Edd }}$ would produce the same scattered surface brightness profile (at $\rho, c t \gg r_{\mathrm{c}}$ ) as a $10^{4}$-year flare at $L_{X}=0.1 L_{\text {Edd }}$.

\subsubsection{Switch-off and switch-on scenarios}

We next consider a scenario in which the central source was persistently (over a time that is longer than the characteristic light travel time of the cluster) bright in the past until it suddenly turned off. A sequence of resulting scattered surface brightness profiles corresponding to different times after the switch-off $(t>0)$ is shown in Fig. 3c. One can see that these profiles differ markedly from those corresponding to the flare case (see Fig. 3b). In particular, as time goes by the surface brightness decreases in the central region, while it remains practically unchanged in more distant parts of the cluster still for a long time $\sim \rho / c$ after the switch-off. This gives rise to a broad $(\delta \rho \sim \rho)$ maximum of the scattered/intrinsic brightness ratio, which propagates outward at an apparent speed of light.

Another interesting situation takes place when the central source is bright at the time of observation but it has turned on recently. This "switch-on" case may actually correspond to the observed quasars, which may have been luminous for a time short compared to the light travel time of the gas around them. This situation is depicted in Fig. 3d. One can see that the radial distribution of scattered AGN radiation is effectively truncated at a certain radius $\sim c\left(t-t_{\mathrm{on}}\right)$. This gives the possibility to estimate the time during which the quasar has been active. 


\subsection{Resonance lines}

The results obtained above can be directly extended to the case of resonance lines provided the intracluster gas is optically thin $(\tau \ll 1)$ to resonant scattering. We shall consider this limit below and then (in Sect. 3.3.1) point out differences that may arise when $\tau \gtrsim 1$.

In the absence of external illumination, the equivalent width of a line would be

$$
\begin{aligned}
E W_{0} & =\int_{-\infty}^{\infty} \epsilon_{\text {line }} \mathrm{d} z / \int_{-\infty}^{\infty} \epsilon_{E, \text { cont }}\left(E_{i k}\right) \mathrm{d} z \\
& =1.2 \times 10^{5} \frac{n_{z}(T)}{n_{\mathrm{e}}} f_{i k} \frac{g_{i k}(T)}{g_{\mathrm{B}}\left(T, E_{i k}\right)}(\mathrm{keV}),
\end{aligned}
$$

under our assumptions of constant temperature and element abundances.

Resonant scattering of the AGN photons leads to an increase of $E W$. At the same time, Thomson scattering of AGN radiation tends to enhance the continuum near the line and thus to decrease its equivalent width. As we know from Sect. 2, the first effect is a factor of $R \sim 6$ larger than the second, hence the net effect of the scattering of AGN emission in the intracluster gas should be increased equivalent widths of the resonance lines. We can then write:

$$
\begin{aligned}
E W= & \left(\int_{-\infty}^{\infty} \epsilon_{\text {line }} \mathrm{d} z+\int_{z_{1}}^{z_{2}} \epsilon_{\text {line }}^{\text {scat }} \mathrm{d} z\right) / \\
& \left(\int_{-\infty}^{\infty} \epsilon_{E, \text { cont }} \mathrm{d} z+\int_{z_{1}}^{z_{2}} \epsilon_{E, \text { cont }}^{\text {scat }} \mathrm{d} z\right) \\
= & E W_{0} \frac{1+R \int_{z_{1}}^{z_{2}} \epsilon_{E, \text { cont }}^{\text {scat }} \mathrm{d} z / \int_{-\infty}^{\infty} \epsilon_{E, \text { cont }} \mathrm{d} z}{1+\int_{z_{1}}^{z_{2}} \epsilon_{E, \text { cont }}^{\text {scat }} \mathrm{d} z / \int_{-\infty}^{\infty} \epsilon_{E, \text { cont }} \mathrm{d} z}
\end{aligned}
$$

If the contribution of scattered AGN radiation to the surface brightness of the cluster is small, the fractional increase of the equivalent line width will be

$$
\begin{aligned}
\frac{\Delta E W}{E W_{0}} & \equiv \frac{E W-E W_{0}}{E W_{0}} \approx \\
& =\frac{R-1}{5} \frac{\int_{z_{1}}^{z_{2}} \epsilon_{E, \mathrm{cont}}^{\text {scat }}\left(E_{i k}\right) \mathrm{d} z}{\int_{-\infty}^{\infty} \epsilon_{E, \mathrm{cont}}\left(E_{i k}\right) \mathrm{d} z} .
\end{aligned}
$$

Using Eq. (26), one can readily apply all the results of Sect. 3.2 to optically thin resonance lines.

It should be emphasized that Eqs. (26) is strictly valid only in the case where both the plasma temperature (which affects the ionization balance and plasma emissivity) and the element abundances are constant along the line of sight corresponding to a given projected distance $\rho$. Indeed, only when these conditions are met, $\epsilon_{\text {line }}(r) \propto \epsilon_{\text {cont }}(r)$ and $\epsilon_{\text {line }}^{\text {scat }}(r) \propto \epsilon_{\text {cont }}^{\text {scat }}(r)$ - see Eqs. (1)-(4). In the general case, a coefficient somewhat different than $R-1$ will relate the ratios $\triangle E W / E W_{0}$ and $\int_{z_{1}}^{z_{2}} \epsilon_{E, \text { cont }}^{\text {scat }} \mathrm{d} z / \int_{-\infty}^{\infty} \epsilon_{E, \text { cont }} \mathrm{d} z$.

We should also remind the reader that we ignore throughout the effect of the AGN on the ionization balance $\left(n_{z}\right)$ in the intracluster medium. The irradiation of the gas by a very bright central source will first of all alter the equivalent width of the emission line relative to the value predicted by Eq. (24); it will also affect (however to a smaller degree, because both $\epsilon_{\text {line }}$ and $\epsilon_{\text {line }}^{\text {scat }}$ are proportional to $n_{z}$ ) the ratio $\Delta E W / E W_{0}$.

\subsubsection{Finite depth effects}

The intracluster gas may be moderately optically thick in the stronger X-ray lines ( $\tau \gtrsim 1$, e.g. Gilfanov et al. 1987b), so the analytic results obtained above will be only approximately valid in that case.

The optical depth at the center of a resonance line along a given direction $\boldsymbol{l}$ through the cluster is

$\tau_{0}=\int \sigma_{0} n_{z}(r) \mathrm{d} l$

At plasma temperatures typical of galaxy clusters $(k T \sim 1-$ $10 \mathrm{keV}$ ), all interesting X-ray lines have nearly Doppler absorption profiles whose width is determined by the velocities of thermal and turbulent motions, because the line natural width is relatively small. Therefore, the cross section at line center is

$\sigma_{0}=\frac{\sqrt{\pi} h r_{\mathrm{e}} c f_{i k}}{\Delta E_{\mathrm{D}}}$

( $h$ is Planck's constant), with the Doppler width being

$$
\begin{aligned}
\Delta E_{\mathrm{D}} & =E_{i k}\left(\frac{2 k T}{A m_{\mathrm{p}} c^{2}}+\frac{V_{\text {turb }}^{2}}{c^{2}}\right)^{1 / 2} \\
& =E_{i k}\left[\frac{2 k T}{A m_{\mathrm{p}} c^{2}}\left(1+1.4 A M^{2}\right)\right]^{1 / 2},
\end{aligned}
$$

where $A$ is the element atomic mass, $m_{\mathrm{p}}$ is the proton mass, $V_{\text {turb }}$ is the characteristic turbulent velocity and $M$ is the corresponding Mach number. The role of the additional line broadening due to turbulence becomes more important with increasing $A$.

Taking into account turbulence line broadening is important for two reasons. First, this phenomenon may cause some resonance lines, which would otherwise have $\tau \gtrsim 1$, to become optically thin. Secondly, some satellite lines may fall within the core of the resonance line, thereby effectively reducing the factor $R$ defined in Eq. (7). As was noted in Sect. 2, this effect is particularly important for the $\mathrm{K} \alpha$ lines of $\mathrm{H}$-like and He-like iron at $k T \sim 1 \mathrm{keV}$.

If $\tau_{0}$ is non-negligible, first of all the intrinsic surface brightness profile of the intracluster gas will be distorted due to diffusion of photons from the central region to the outer parts of the cluster. The equivalent line width will be somewhat smaller than predicted by Eq. (24) at $\rho \lesssim r_{\mathrm{c}}$, and larger at $\rho \gtrsim r_{\mathrm{c}}$ (Gilfanov et al. 1987b). Note that this effect takes place already at $\tau_{0}<1$, when only single resonant scattering is important, and becomes more pronounced when $\tau_{0}>1$.

When the optical depth becomes larger than unity, multiple resonant scatterings come into play. In the stationary scenario, the surface brightness profile of scattered AGN emission in a line with $\tau_{0} \gtrsim 1$ will be flatter in the cluster core compared to a $1 / \rho$ profile in the $\tau_{0} \ll 1$ case. Also, the total flux of AGN photons scattered toward us from the core region will be smaller than calculated in the single-scattering approximation by a factor $\sim \tau_{0}$. However, due to the rather similar effects taking place for thermal gas emission (see above), the net correction to the ratio $E W / E W_{0}$ is expected to be fairly small for $\tau_{0} \gtrsim 1$. 
Multiple resonant scatterings also delay the escape of AGN photons from the cluster core. As a result, the time-dependent scattered surface brightness profiles arising in the outburst scenario will be affected.

\section{Numerical simulations}

As a next step, we have performed numerical simulations for two actual clusters. We used a combination of 1) a MonteCarlo code to play the diffusion of photons (both those emitted by the AGN and by the gas) through multiple resonant scatterings and 2) the MEKAL code (Kaastra 1992) as implemented in the software package XSPEC v10 (Arnaud 1996) to calculate plasma emissivity in lines. The line energies and oscillator strengths were taken from the list of strong resonance lines of Verner et al. (1996) and the solar abundances of elements from Anders \& Grevesse (1989). The ionization fractions were calculated using collisional ionization rate fits from Voronov (1997), radiative recombination rates from Verner \& Ferland (1996) and dielectronic recombination rates from Aldrovandi \& Pequignot (1973), Shull \& van Steenberg (1982) and Arnaud \& Rothenflug (1985).

\subsection{87/Virgo}

One of the most promising targets in the sky for observations of the discussed effect is M 87. This is a giant elliptical galaxy located near the center of the nearest $(17 \mathrm{Mpc}-1$ arcmin corresponds to $5 \mathrm{kpc}$ ) rich cluster of galaxies Virgo. M 87 hosts a central black hole of mass $3 \times 10^{9} M_{\odot}$ (Macchetto et al. 1997; Marconi et al. 1997), which is the largest reliably measured black hole mass. The corresponding Eddington luminosity $L_{\text {Edd }}=4 \times 10^{47} \mathrm{erg} / \mathrm{s}$.

M 87 demonstrates AGN activity. In particular, observations at different energy bands reveal an unresolved nucleus and a one-sided jet. The combined bolometric luminosity of the nucleus and jet is $\sim 10^{42} \mathrm{erg} \mathrm{s}^{-1}$ (e.g. Biretta et al. 1991), or $\sim 10^{-6}$ of the Eddington luminosity. This is an amazingly small value given the fact that the nucleus is located in the center of a dense cooling flow. The average power input in the form of relativistic plasma from the jet into a large-scale radio halo around M 87 is estimated (Owen et al. 2000) to have been $\sim 10^{44} \mathrm{erg} \mathrm{s}^{-1}$ over the past $\sim 10^{8}$ years, which is still a small fraction of the Eddington luminosity.

Both the nucleus and the jet (whose brightest knot is at a distance of $\sim 1 \mathrm{kpc}$ from the nucleus) have recently been observed in X-rays with the XMM-Newton (Böhringer et al. 2001) and Chandra (Wilson \& Yang 2002) satellites. The $\mathrm{X}$-ray spectrum is power-law with values for the photon index ranging between 2 and 2.9 for the nucleus and different knots in the jet. The inferred X-ray (1-10 keV) luminosities of the nucleus and the jet are $\sim 3 \times 10^{40}$ and $8 \times 10^{40} \mathrm{erg}$, respectively (Böhringer et al. 2001).

In our simulations, we modeled the distribution of intracluster gas around M 87 based on the results of recent analyses of the XMM observations by Böhringer et al. (2001), Finoguenov et al. (2002) and Matsushita et al. (2002). We assumed the distribution of gas to be spherically symmetric
Table 1. The brightest X-ray and extreme UV resonance lines of the M 87/Virgo intracluster gas.

\begin{tabular}{lccc}
\hline \hline Ion & $\begin{array}{c}\text { Energy } \\
(\mathrm{keV})\end{array}$ & $\begin{array}{c}\text { Equivalent width } \\
(\mathrm{eV})\end{array}$ & Optical depth \\
\hline Fe XXIV & 0.049 & 1 & 3.0 \\
Fe XXIV & 0.065 & 2 & 6.0 \\
Fe XXIII & 0.093 & 3 & 8.5 \\
O VIII & 0.654 & 20 & 0.6 \\
Ne X & 1.021 & 30 & 0.9 \\
Fe XXIII & 1.129 & 10 & 3.2 \\
Fe XXIV & 1.166 & 30 & 2.7 \\
Si XIII & 1.865 & 10 & 1.3 \\
Si XIV & 2.005 & 60 & 1.3 \\
S XV & 2.461 & 20 & 1.3 \\
Ar XVII & 3.140 & 20 & 0.4 \\
Fe XXV & 6.700 & 740 & 1.2 \\
Fe XXV & 7.881 & 110 & 0.2 \\
\hline
\end{tabular}

around M 87 and restricted our consideration to the central region with a radius of $250 \mathrm{kpc}$. The electron density radial profile was represented by a sum of two beta-models (9) with the following parameters: $n_{0}=0.13 \mathrm{~cm}^{-3}, r_{\mathrm{c}}=1.7 \mathrm{kpc}$, $\beta=0.42$ and $n_{0}=0.011 \mathrm{~cm}^{-3}, r_{\mathrm{c}}=22 \mathrm{kpc}, \beta=0.47$. We adopted a constant temperature $k T=1 \mathrm{keV}$ within the central $1 \mathrm{kpc}$, the dependence $k T=1.5(r / 5 \mathrm{kpc})^{0.22} \mathrm{keV}$ between 1 and $50 \mathrm{kpc}$, and a constant $k T=2.5 \mathrm{keV}$ at larger radii. The last value approximately corresponds to temperatures measured with ASCA (Shibata et al. 2001) in different parts of the Virgo cluster within $\sim 300 \mathrm{kpc}$ from M 87. The element abundances were taken to be constant at $r<10 \mathrm{kpc}$, with $A(\mathrm{O})=0.4, A(\mathrm{Si})=1.0, A(\mathrm{~S})=1.0, A(\mathrm{Ar})=1.0$ and $A(\mathrm{Fe})=0.8$ (in units of solar abundances), then gradually declining to $A(\mathrm{O})=0.4, A(\mathrm{Si})=0.6, A(\mathrm{~S})=0.6, A(\mathrm{Ar})=0.6$ and $A(\mathrm{Fe})=0.4$ at $r=40 \mathrm{kpc}$, and constant thereafter.

Table 1 lists resonance X-ray and extreme UV lines that we estimate to be the strongest emission lines of the M 87 gas. All of these lines have substantial optical depths from the center to the boundary of the gas cloud, assuming negligible turbulence.

Using Eq. (20), we can estimate for the stationary scenario the contribution of scattered AGN radiation to the surface brightness of M 87/Virgo in the X-ray continuum:

$$
\begin{aligned}
\frac{B_{E, \text { cont }}^{\text {scat }}}{B_{E, \text { cont }}}= & 4 \times 10^{2} \frac{L_{X}}{L_{\mathrm{Edd}}} E^{-0.6} \exp (E / 1.5 \mathrm{keV}) \\
& \times\left(\frac{\rho}{1.7 \mathrm{kpc}}\right)^{-0.7}, 1.7 \mathrm{kpc} \ll \rho \ll 22 \mathrm{kpc} ;
\end{aligned}
$$

$$
\begin{aligned}
\frac{B_{E, \text { cont }}^{\text {scat }}}{B_{E, \text { cont }}}= & 40 \frac{L_{X}}{L_{\mathrm{Edd}}} E^{-0.6} \exp (E / 2 \mathrm{keV}) \\
& \times\left(\frac{\rho}{22 \mathrm{kpc}}\right)^{-0.6}, \rho \gg 22 \mathrm{kpc} .
\end{aligned}
$$

Here we have assumed constant temperatures of $k T=1.5 \mathrm{keV}$ and $k T=2 \mathrm{keV}$ for Eqs. (30) and (31), respectively, and $\gamma=2$.

The corresponding dependences for the equivalent width of a resonance line with $\tau_{0} \ll 1$ can be found from Eqs. (30) 


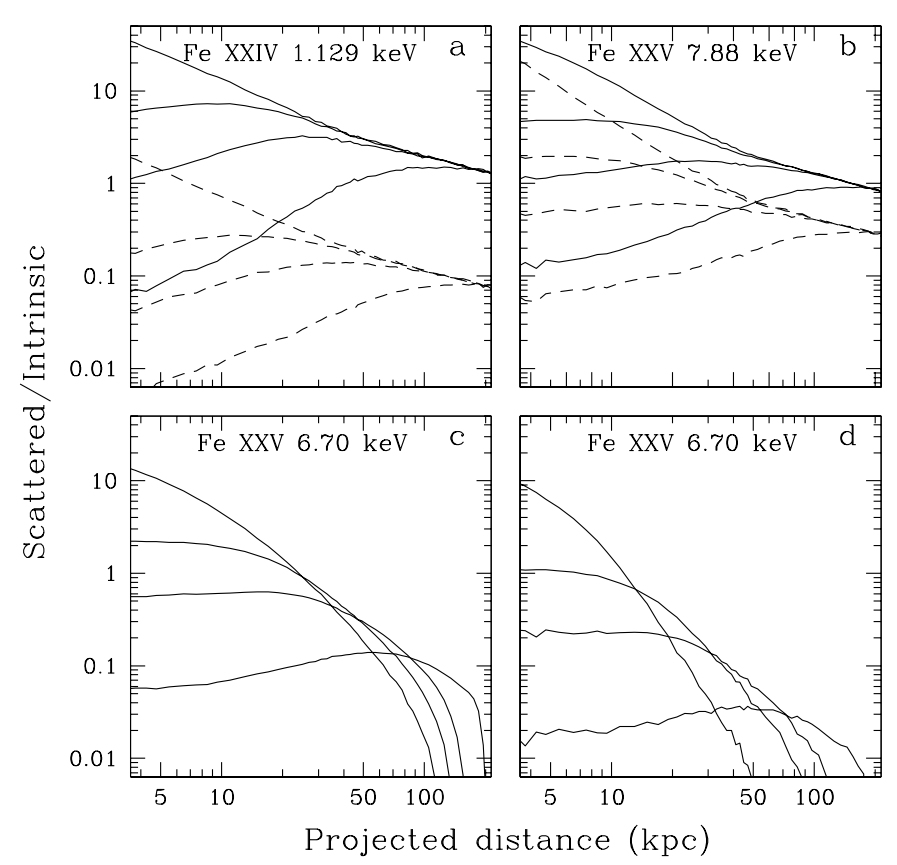

Fig. 5. a) Ratio of the surface brightness of scattered AGN radiation to the intrinsic brightness of the hot gas in the iron $1.13 \mathrm{keV}$ resonance line, in the switch-off scenario for M 87, at different times after AGN switch-off as a function of projected radius. The AGN emitted $L_{X}=$ $0.01 L_{\mathrm{Edd}}$ in the past. The different solid lines correspond, from top to bottom, to elapsed times of 5, 50, 100 and 250 thousand years. The dashed lines are the corresponding profiles for the spectral continuum at the energy of the resonance line. b) Same as Fig. 1a, but for the iron $\mathrm{Fe} 7.88 \mathrm{keV}$ line. c) Same as a), but for the iron $6.70 \mathrm{keV}$ line and a $10^{5}$-year outburst $\left(L_{X}=0.01 L_{\text {Edd }}\right)$. d) Same as c), but for a $2 \times 10^{4}$-year outburst.

and (31) as $\Delta E W / E W_{0}=(R-1) B_{E, \text { cont }}^{\text {scat }}\left(E_{i k}\right) / B_{E \text {,cont }}\left(E_{i k}\right)$ (assuming constant element abundances for a given $\rho$ ).

Now we proceed to discussing the results of our computations, which do not use the assumption of small optical depth.

\subsubsection{Switch-off scenario}

We first consider a scenario in which M 87 was bright in the past for a long time (at least $\sim$ a million years) until it suddenly turned off (actually switched to its present low-luminosity state) at $t_{\text {off }}=0$. We have plotted in Figs. 5a and b for two resonance lines the computed contribution of scattered radiation from the AGN to the surface brightness as a function of projected radius as would be measured by observers living in various epochs $t>0$ after the switch-off. Also shown are the corresponding profiles for the continuum emission near the lines. The relative contribution of scattered AGN emission is a factor of $R \sim 6$ (with a scatter of $\sim 3$ between the lines) larger for the lines than for the continuum, in agreement with the result of Sect. 2. We adopted $L_{X}=0.01 L_{\text {Edd }}$ for our examples. Since the discussed effect is proportional to $L_{X}$, the results can be easily recomputed for any AGN luminosity.

Figure 6 compares the surface brightness profiles of scattered AGN radiation and thermal emission in the iron $6.7 \mathrm{keV}$ line, which has the largest equivalent width in our sample. It is

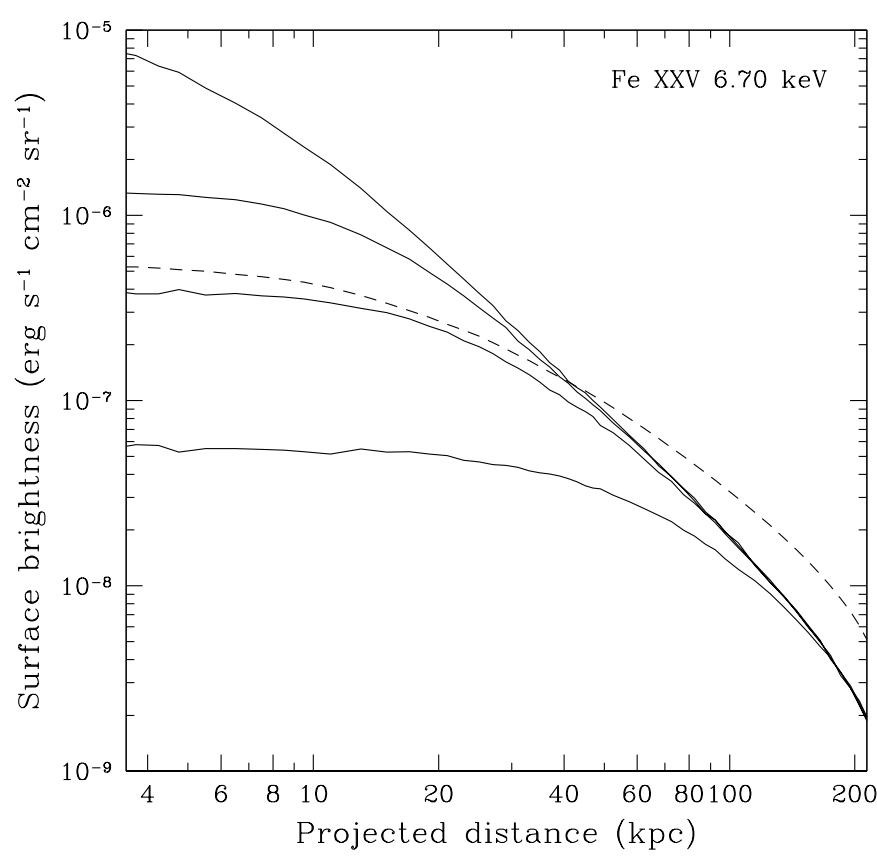

Fig. 6. M 87/Virgo surface brightness of scattered AGN radiation (solid lines) in the iron $6.70 \mathrm{keV}$ resonance line at different times (as in Fig. 5) after the AGN switch-off. The dashed line shows the intrinsic surface brightness profile of the hot gas in the $6.70 \mathrm{keV}$ line, which also is distorted by resonant scattering. The AGN emitted $L_{X}=0.01 L_{\text {Edd }}$ in the past.

essential that photon statistics is not expected to impose strong restrictions on the prospects of detecting scattered AGN radiation from the Virgo cluster out to $\sim 1 \mathrm{Mpc}$ from M 87 with the next generation of $\mathrm{X}$-ray spectrometers.

The finite depth effects that were discussed in Sect. 3.3.1 play in the M 87 case only a minor role for the X-ray lines, because for them $\tau_{0}<3$ (see Table 1). Figure 7 compares two cases, $M=0$ (no turbulence) and $M=0.5$ (significant turbulence), for the iron $1.13 \mathrm{keV}$ line. The increased turbulence leads to a decrease from $\tau_{0}=3.2$ to $\tau_{0}=0.7$. We see that the relative contribution of scattered AGN emission to the surface brightness in the central $\sim 10 \mathrm{kpc}$ is several times larger in the former (optically-thick) case, except for the early moments. This increase is mainly due to the delayed escape of photons.

We can now address the principal question of our study: what constraints can X-ray observations place on the past luminosity of the M 87 central source? Suppose that it is possible in an experiment to map the distribution of the equivalent width of a particular line over M 87/Virgo with $\delta=10 \%$ accuracy, which we consider a realistic value (see the discussion in Sect. 5). Should then the relative contribution of scattered AGN emission exceed $\delta$ at least at some projected radius (i.e. at least at the maximum of $E W / E W_{0}$ in Fig. 5), it will be possible to estimate the past AGN luminosity. The resulting dependence of the minimum detectable past luminosity of M 87 on time after the switch-off is plotted in Fig. $8 \mathrm{a}$.

We see that in order to have a $10 \%$ contribution of scattered radiation from the AGN whose luminosity in the past was $L_{X}=10^{-4} L_{\text {Edd }}=4 \times 10^{43} \mathrm{erg} \mathrm{s}^{-1}$ (i.e. several hundred times higher than the combined X-ray luminosity of the nucleus and 


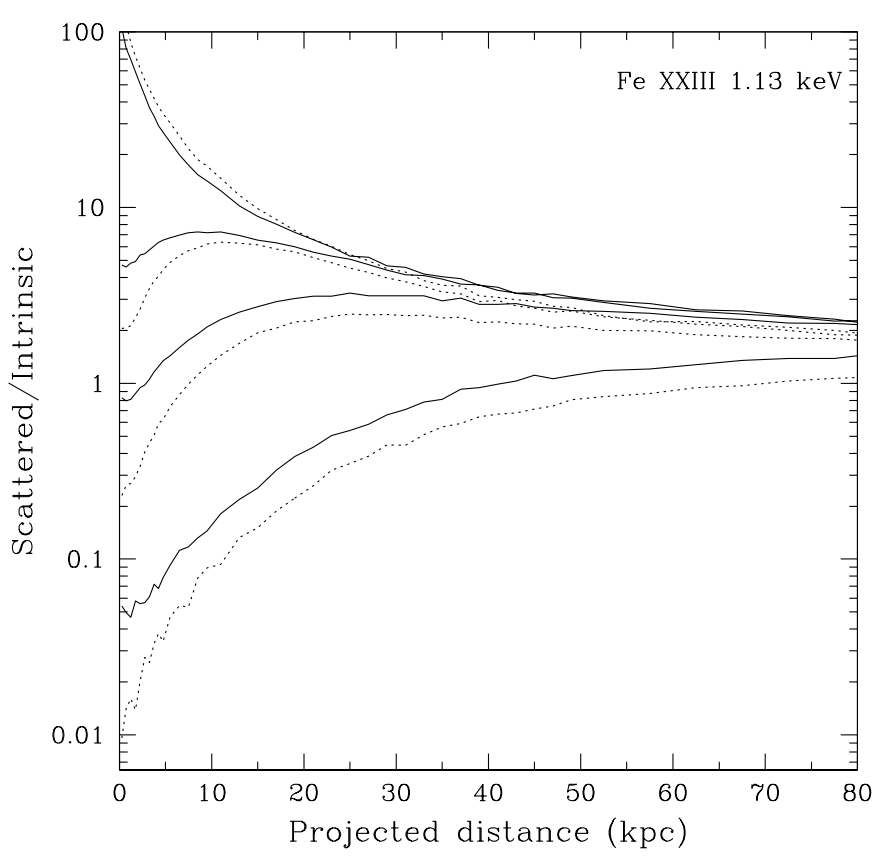

Fig. 7. Ratio of the surface brightness of scattered AGN radiation to the intrinsic brightness of the hot gas in the iron $1.13 \mathrm{keV}$ resonance line, in the switch-off scenario for M 87, as a function of projected radius. The sampled times are as in Fig. 5. The solid lines correspond to the case of negligible turbulence, and the dotted lines to $M=0.5$. Note the linear scaling chosen for the projected radius, as opposed to the logarithmic scaling in Fig. 5.

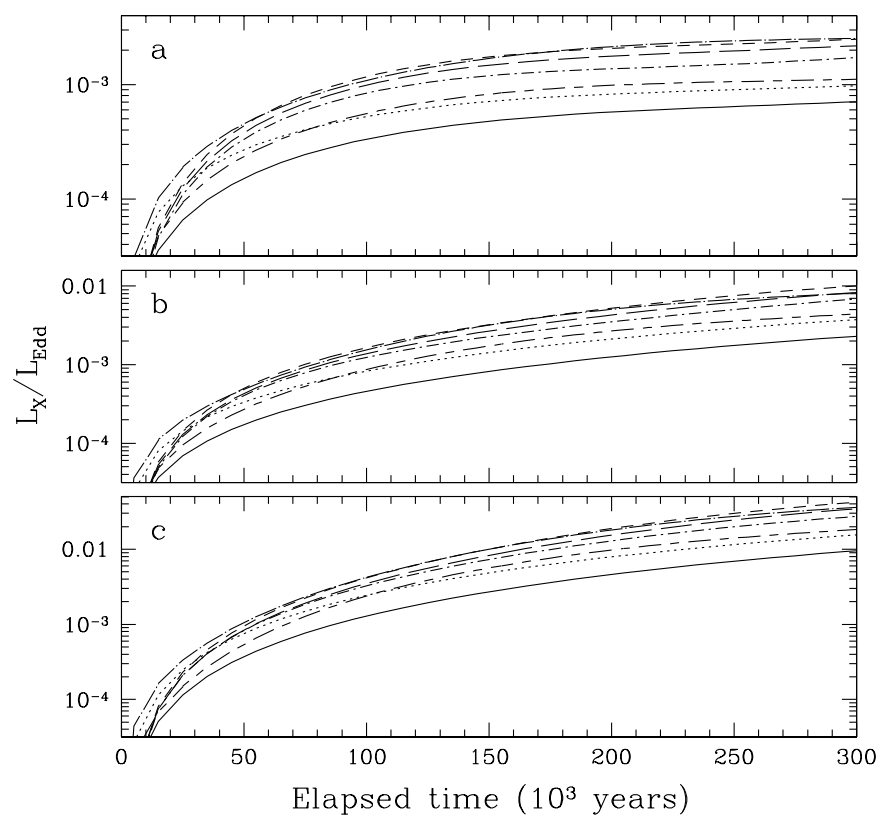

Fig. 8. a) Minimum detectable (for $\delta=10 \%$ - see text) past X-ray luminosity as a function of elapsed time in the switch-off scenario for M 87. The different curves correspond to mapping M 87/Virgo in different lines: Fe $1.13 \mathrm{keV}$ line (solid), Fe $1.17 \mathrm{keV}$ line (dotted), Si $1.86 \mathrm{keV}$ line (short-dashed), S $2.46 \mathrm{keV}$ line (long-dashed), Ar $3.14 \mathrm{keV}$ line (dot-short-dashed), Fe $6.70 \mathrm{keV}$ line (dot-long-dashed) and $\mathrm{Fe} 7.88 \mathrm{keV}$ line (short-dash-long-dashed). b) Same as (a), but for a $10^{5}$-year flare. c) Same as b), but for a $2 \times 10^{4}$-year flare.

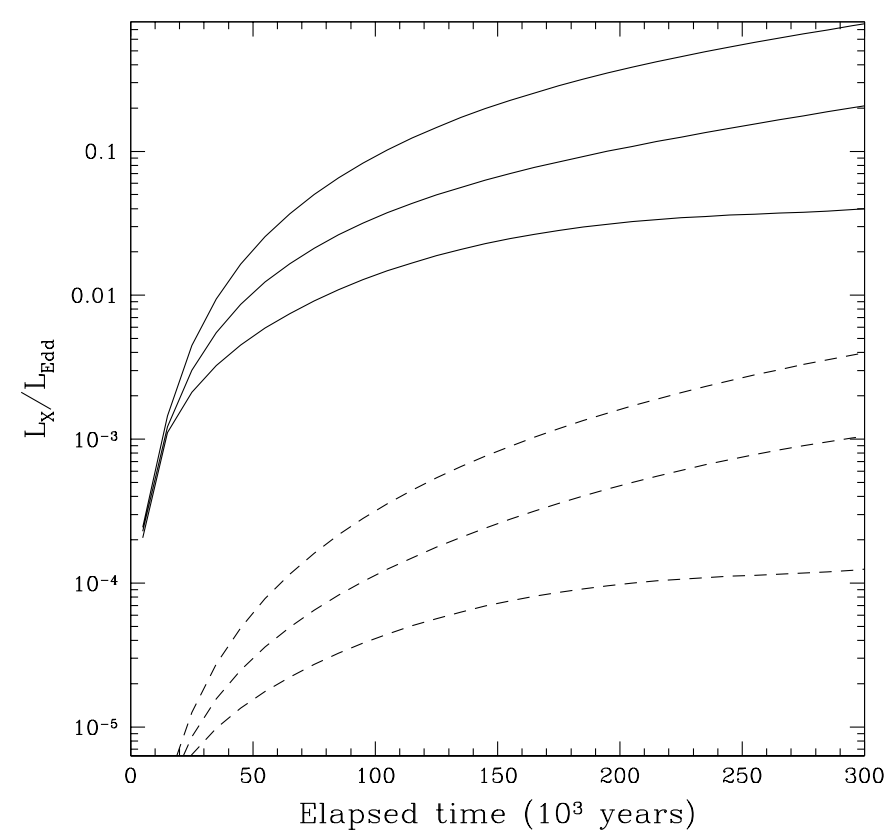

Fig. 9. Upper limits (for $\delta=100 \%$ ) on the past X-ray luminosity of the M 87 AGN as a function of elapsed time, derived from XMM-Newton observations of the M 87/Virgo central region in the $\mathrm{X}$-ray spectral continuum below $7 \mathrm{keV}$, for three cases: switch-off, a $10^{5}$-year flare and a $2 \times 10^{4}$-year flare (solid lines from bottom to top). For comparison shown (dashed lines) are the limits obtainable if it were possible to map M 87/Virgo at $E=20 \mathrm{keV}$.

jet now), we should be not later than $\sim 3 \times 10^{4}$ years after the switch-off and look with X-ray telescopes into the M 87 core region $(\rho \lesssim 10 \mathrm{kpc} \sim 2$ arcmin in the plane of the sky), as is clear from Fig. 5. If the luminosity in the past was higher, $L_{X}=10^{-3} L_{\text {Edd }}=4 \times 10^{44} \mathrm{erg} \mathrm{s}^{-1}$, we must live not later than $\sim 2 \times 10^{5}$ years after the switch-off and look further out (at $\rho \sim 50-200 \mathrm{kpc} \sim 20$ arcmin from the AGN). The nearly flat $\left(\propto t^{-0.6}\right)$ shape of the curves in Fig. 8a, which have been truncated at $t=3 \times 10^{5}$ years to mimimize the effects of the finite size of our model gas cloud, suggests that if the X-ray luminosity of M 87 was $\sim$ a few $10^{-3} L_{\text {Edd }} \sim 10^{45} \mathrm{erg} \mathrm{s}^{-1}$ in the past, the maximum allowed elapsed time would only be restricted by the extent of the gas in the Virgo cluster $(\sim 1 \mathrm{Mpc})$, the effective area of the detector and the field of view of the telescope.

We may therefore conclude that it should be possible with future X-ray spectrometers to measure or obtain fairly tight limits on the past X-ray luminosity of the central source in M 87. For comparison, we can use the available data of XMMNewton observations of the M 87/Virgo core to derive some weaker limits already now. Böhringer et al. (2001) have analyzed X-ray spectra taken from a sequence of rings centered on the M 87 nucleus. The most distant ring has an outer radius $r_{\text {out }} \approx 60 \mathrm{kpc}$, and the innermost circle has $r_{\text {in }} \approx 1.25 \mathrm{kpc}$. Except for the spectrum of the central circle to which the direct emission from the AGN contributes, the individual spectra correspond to optically thin thermal emission from the gas. The quality of the spectra is apparently good up to $\sim 7 \mathrm{keV}$. We can therefore suggest that the surface brightness of scattered AGN radiation (having a power-law spectrum) does not exceed the 
actually measured surface brightness in the X-ray continuum at $7 \mathrm{keV}$ nowhere between $\rho=1.25 \mathrm{kpc}$ and $60 \mathrm{kpc}$. From this condition we can find an upper limit on the past X-ray luminosity of M 87 as a function of time after the switch-off, similarly as we derived the limits presented in Fig. 8a based on the resonance line computations. However, in the previous case we did not impose any limitations on the projected radius and we adopted a value $\delta=10 \%$, rather than $\delta=100 \%$ to be used now. The resulting dependence is shown in Fig. 9.

We see that the current limits based on the spectral continuum data are rather weak. For example, the average X-ray luminosity of the M 87 AGN over a long period of $\gtrsim 10^{5}$ years ending $5 \times 10^{4}$ years ago was not higher than $5 \times 10^{-3} L_{\mathrm{Edd}}=$ $2 \times 10^{45} \mathrm{erg} \mathrm{s}^{-1}$. By comparing Fig. 9 with Fig. 8a, we see that the current limits could be improved by at least an order of magnitude by means of fine X-ray spectroscopy. Alternatively, much tighter limits could be obtained by measuring the surface brightness of M 87/Virgo in the hard X-ray continuum. To illustrate this possibility, we have repeated our analysis for $E=20 \mathrm{keV}$ - see the result in Fig. 9. Going from $E=7 \mathrm{keV}$ to $20 \mathrm{keV}$ (again assuming a $\delta=100 \%$ scattered contribution relative to the bremsstrahlung emission) leads to a factor of $\sim 200$ (!) stronger limits.

\subsubsection{Flare scenario}

Suppose now that some time ago M 87 experienced an outburst that was short compared to the characteristic light crossing time of the gas cloud, i.e. $\Delta \ll 10^{6}$ years. Some analytic estimates for this case were obtained in Sect. 3.2.3. Figures 5c and d show the computed time evolution of the scattered/thermal brightness ratio profile for the $\mathrm{Fe} X \mathrm{XV} \mathrm{K} \alpha$ line for outburst durations $\Delta=10^{5}$ years and $\Delta=2 \times 10^{4}$ years, respectively, and an AGN luminosity $L_{X}=0.01 L_{\text {Edd }}$. Note that for $t \gg \Delta$ the scattered surface brightness profile depends on the product $L_{X} \Delta$, rather than on $L_{X}$ and $\Delta$ separately.

Figures $8 \mathrm{~b}$ and $8 \mathrm{c}$ show the minimum detectable (for $10 \%$ scattered contribution) AGN outburst luminosity as a function of elapsed time for the two considered scenarios. These dependences should be compared with Fig. 8a representing the switch-off scenario. We see that until a certain moment $t_{\text {crit }} \sim \Delta$ the minimum detectable luminosity is only a little smaller in the flare case than in the switch-off case. This is expected, because the characteristic line-of-sight depth of the illuminated volume at $t=t_{\text {crit }}$ is about the same, $\delta \rho \sim c t_{\text {crit }}$, in both cases. Only at $t \gg \Delta$, does the minimum detectable luminosity become much larger (by a factor of $\Delta / t$ ) for short outbursts. This directly follows from comparison of Eqs. (20) and (23).

Figure 9 shows upper limits for the same outburst scenarios but obtained using the XMM-Newton data on the X-ray continuum emission from M 87/Virgo.

\subsection{Cygnus $A$}

Our second example is Cyg A. This is a well-known nearby $(z=0.0562$, i.e. about 20 times more distant than M 87 1 arcmin approximately corresponds to $100 \mathrm{kpc}$ ) powerful
Table 2. The brightest resonance X-ray and extreme UV lines of the Cyg A intracluster gas.

\begin{tabular}{lccc}
\hline \hline Ion & $\begin{array}{c}\text { Energy } \\
(\mathrm{keV})\end{array}$ & $\begin{array}{c}\text { Equivalent width } \\
(\mathrm{eV})\end{array}$ & Optical depth \\
\hline Fe XXIV & 0.049 & 1 & 0.9 \\
Fe XXIV & 0.065 & 2 & 1.8 \\
Fe XXV & 6.70 & 250 & 1.8 \\
Fe XXV & 7.88 & 40 & 0.3 \\
Fe XXVI & 6.97 & 200 & 0.3 \\
\hline
\end{tabular}

radio galaxy. Cyg A has recently been observed with the Chandra satellite, and detailed information was obtained on the morphology of X-ray emission from the galaxy (Young et al. 2002) as well on the intracluster gas surrounding it (Smith et al. 2002).

In the Chandra and quasi-simultaneous RXTE observations (Young et al. 2002), hard (up to $100 \mathrm{keV}$ ), spatially unresolved $\mathrm{X}$-ray emission was detected from the position of the radio and infrared nucleus of Cyg A. The energy spectrum of this radiation is power law with photon index $\gamma=1.5$, heavily absorbed below a few keV. The inferred unabsorbed 1-10 keV luminosity of the nucleus $L_{X} \sim 5 \times 10^{44} \mathrm{erg} \mathrm{s}^{-1}$. This is four orders of magnitude more than the X-ray luminosity of the M 87 nucleus.

The Chandra observations clearly reveal an intracluster medium. The gas has complex structure within the central $\sim 100 \mathrm{kpc}$, which apparently is the result of an interaction with the relativistic material produced by AGN activity. In contrast, the morphology of the gas is simple at larger radii ( $\gtrsim 100 \mathrm{kpc})$, namely the gas appears to be spherical within at least $700 \mathrm{kpc}$ of the nucleus, and nearly isothermal with $k T \sim 7-8 \mathrm{keV}$. The total luminosity of the intracluster gas is $\sim 10^{45} \mathrm{erg} \mathrm{s}^{-1}$, which is comparable to the X-ray luminosity of the central AGN and is a typical value for rich clusters of galaxies.

Given the above observational facts, we can estimate the contribution of scattered emission from the AGN to the X-ray surface brightness outside the central $100 \mathrm{kpc}$ of the Cyg A cluster. Due to the highly irregular distribution of gas in the innermost region of Cyg A, our simulations will not be aimed at this zone. Based on these considerations and the results of Smith et al. (2002), we model the radial distribution of gas density by a beta-model with $n_{0}=0.05 \mathrm{~cm}^{-3}, r_{\mathrm{c}}=30 \mathrm{kpc}$ and $\beta=0.5$. The temperature is assumed to be constant, $k T=5 \mathrm{keV}$, within the central $50 \mathrm{kpc}$ and also at $r>150 \mathrm{kpc}$, $k T=7.5 \mathrm{keV}$. The abundance of iron is taken to be 0.35 solar. Our model cloud of gas has an outer boundary at $r=1 \mathrm{Mpc}$.

Table 2 lists the strongest emission lines of the Cyg A cluster, as implied by our model. The Chandra and RXTE data do reveal two strong lines, one near $6.7 \mathrm{keV}(\mathrm{Fe} \mathrm{K} \alpha)$ and another near $7.9 \mathrm{keV}$ ( $\mathrm{Fe} \mathrm{K} \beta$ plus possibly Ni K $\alpha$ ). It appears (see Fig. 7 in Smith et al. 2002) that the measured (with moderate energy resolution) spectra also do not contradict the presence of a strong line at the position of Fe $\operatorname{Ly} \alpha(6.97 \mathrm{keV})$. 


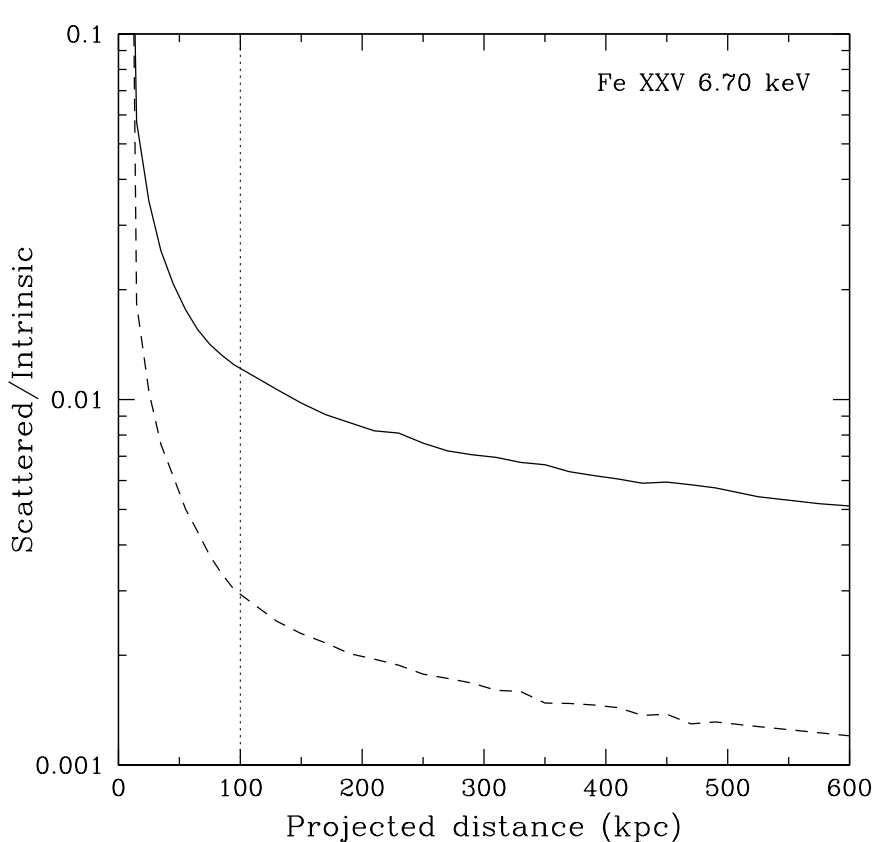

Fig. 10. Ratio of the surface brightness of scattered AGN radiation to the intrinsic brightness of the hot gas in the iron $6.70 \mathrm{keV}$ resonance line, in the stationary scenario for Cyg A, as a function of projected radius (the solid line). The AGN X-ray luminosity is assumed to be equal to its present-day value, $L_{X}=5 \times 10^{44} \mathrm{erg} \mathrm{s}^{-1}$. The dashed line shows the corresponding profile for the spectral continuum at the resonance energy. The vertical dotted line indicates that the results to the left of it are not reliable.

We can estimate from Eq. (20) the stationary contribution of scattered AGN radiation to the surface brightness of the Cyg A cluster in the X-ray continuum:

$$
\begin{aligned}
\frac{B_{E, \text { cont }}^{\text {scat }}}{B_{E, \text { cont }}}= & 2 \times 10^{-3} \frac{L_{X}}{5 \times 10^{44} \mathrm{ergs}^{-1}} E^{-0.1} \exp (E / 7.5 \mathrm{keV}) \\
& \times\left(\frac{\rho}{30 \mathrm{kpc}}\right)^{-0.5}
\end{aligned}
$$

where we have normalized the X-ray luminosity of the Cyg A nucleus to its present-day value and used $\gamma=1.5$.

Figure 10 shows a computed Cyg A radial profile of the scattered/thermal surface brightness ratio for the iron $6.7 \mathrm{keV}$ line, assuming that the AGN X-ray luminosity has remained for a few million years the same as it is now (the stationary scenario). We see that scattered AGN emission contributes of the order of $1 \%$ at $\rho \sim 200 \mathrm{kpc}$. It then follows that the Cyg A luminosity should have been an order of magnitude higher in the past (i.e. $L_{X} \sim 5 \times 10^{45} \mathrm{erg} \mathrm{s}^{-1}$ ) for scattered AGN radiation to contribute $\sim 10 \%$ to the strong $X$-ray lines observed from the intracluster gas (see Fig. 11).

We can also use the available Chandra data on the X-ray continuum emission from the Cyg A cluster to find upper limits on the past X-ray luminosity of its central source. We adopt the following parameters for this analysis: $E=8 \mathrm{keV}$ - the maximum photon energy at which thermal bremsstrahlung emission is still detected, and $\rho_{\min }=10^{2} \mathrm{kpc}$ and $\rho_{\max }=6 \times 10^{2} \mathrm{kpc}-$ the boundaries of the region that is well explored with Chandra. We plot the resulting upper limits in Fig. 11b. We see that the

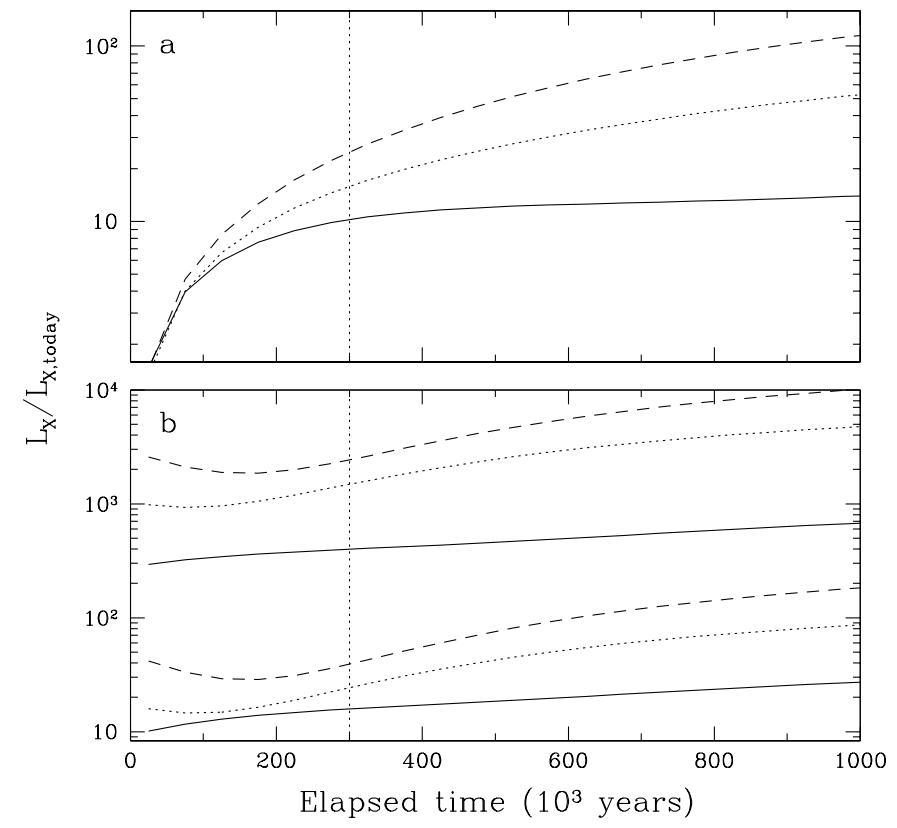

Fig. 11. a) Minimum detectable (for $\delta=10 \%$ - see text) past Xray luminosity of Cyg $\mathrm{A}$ as a function of elapsed time. The surface brightness is measured in the iron $6.70 \mathrm{keV}$ line. The solid line corresponds to the switch-off case $(\Delta \rightarrow \infty)$. The dotted and dashed lines correspond to outbursts of duration $\Delta=5 \times 10^{5}$ years and $\Delta=2 \times 10^{5}$ years, respectively. The luminosity is normalized to the present-day X-ray luminosity of the Cyg A nucleus. The vertical dotted line indicates that the results to the left of it are not reliable. b) Same as a), but derived from Chandra observations of the Cyg A cluster in the X-ray spectral continuum below $8 \mathrm{keV}$ (for $\delta=100 \%$ ). For comparison shown (the lower set of lines) are the limits that could be obtained if it were possible to map the Cyg A cluster at $E=40 \mathrm{keV}$.

current constraints are very weak because of the high temperature $(\sim 7 \mathrm{keV})$ of the intracluster gas. For comparison (see Fig. 11b), arcminute-resolution observations of the Cyg A cluster at $E \sim 40 \mathrm{keV}$ could provide limits on its past luminosity similar to those obtainable by mapping the cluster in resonance lines (see Fig. 11a).

\section{Discussion and conclusions}

The main results of this paper are as follows.

- We have demonstrated that there are two viable observational strategies for constraining the past X-ray luminosity of galactic nuclei with the next generation of X-ray telescopes. One is to search for scattered AGN radiation in the spectral continuum at high energies $E \gg k T(1+z)$, and the other is to perform imaging in bright resonance X-ray lines. The first approach appears to be particularly promising for studying distant (at $z \gtrsim 0.5$ ) powerful quasars and their environments (Sect. 3.2).

- We have shown that the relative contribution of scattered radiation is typically larger by a significant factor of 3-10 in a resonance line than in the neighboring continuum (Sect. 2).

- We have estimated the level of constraints that could be derived from future observations on the past X-ray luminosity of the nearby M 87 and Cyg A active galaxies (Sect. 4). 
The upper limits imposed by the available XMM-Newton and Chandra X-ray continuum data are typically $1-2$ orders of magnitude weaker.

Although we have been mostly discussing clusters of galaxies and their central dominant galaxies, the same method can also be applied to groups of galaxies and isolated giant elliptical galaxies, which also are large reservoirs of hot ionized gas capable of scattering AGN radiation. The main advantage of clusters is the larger extent of intracluster gas, which enables studying previous galactic activity on longer timescales (up to a few times $10^{6}$ years for the richest clusters) than in the case of groups of galaxies and early-type galaxies (up to a few times $10^{5}$ years). On the other hand, the latter two classes of object have their own advantage that typical gas temperatures for them are $k T \sim 1 \mathrm{keV}$ and the factor $\exp (E / k T)$ becomes significant already in the standard X-ray band $(<10 \mathrm{keV})$. In particular, one can use the $\mathrm{K} \alpha$ line of He-like iron at $6.7 \mathrm{keV}$. Indeed, the corresponding ions (Fe XXV) are still abundant at such low temperatures, but collisional excitation of the ions leading to emission in the line is already inefficient.

Although we have only considered the case where the AGN is an isotropic source, in reality angular anisotropy at some level is expected. Therefore, the contribution of scattered emission from the AGN to the surface brightness may be larger or smaller in some regions than predicted assuming source isotropy. We refer the reader to the papers by Gilfanov et al. (1987a) and Wise \& Sarazin (1992) for a detailed discussion of scattered surface brightness profiles that may arise in the case of beamed source emission.

In the discussion of our simulations for the M 87 and Cyg A galaxies and their associated clusters of galaxies we used a fiducial value of $10 \%$ as the minimum detectable contribution of scattered AGN emission to a resonance line. We realize that significant efforts will be required to achieve this or better detection level experimentally. One difficulty is that the measured surface brightness is the integral of emission (thermal plus scattered) along a given line of sight, and there may be present gas with different temperatures and element abundances along this line of sight. Both types of variations will have an effect on the equivalent line width. In principle it should be possible to determine and subtract the underlying temperature and abundance radial trends from a careful analysis of the spectralimaging data. The remaining uncertainties are expected to be largest for cluster central regions, where both the gas temperature and element abundances vary significantly, for example in the M 87 case. On the other hand, this problem is not expected to be severe outside cluster cores. For example, in the Cyg A cluster the gas appears to be nearly isotropic outside the central $\sim 200 \mathrm{kpc}$ (see Smith et al. 2002), whereas the equivalent width of the $6.7 \mathrm{keV}$ iron emission line changes by only $\sim 10 \%$ when the gas temperature varies by $0.5 \mathrm{keV}$ around $k T \sim 7.5 \mathrm{keV}$. Therefore, achieving the $10 \%$ detection level appears realistic.

It is clear that the potential of the method can be fully realized only when it is possible to resolve the interesting resonance lines from neighbouring lines. Indeed, lines other than resonance lines can contribute significantly to the intrinsic emission of the gas, but little to the scattered AGN emission.
Moreover, it is desirable that all important lines in blends be resolved, such as the permitted, intercombination and forbidden lines in the complex around the $6.7 \mathrm{keV}(\mathrm{Fe} \mathrm{XXV} \mathrm{K} \alpha)$ line. Even a small contribution of scattered emission from the AGN could then be made manifest by comparing the surface brightness profiles measured in these lines. We are therefore looking forward to future high-energy astrophysics missions such as Astro-E2, Constellation-X and XEUS that will provide the required energy resolution $(\sim \mathrm{a}$ few $\mathrm{eV})$.

Finally, we would like to note that the constraints on the past AGN X-ray luminosity could be further improved by means of X-ray polarimetry, especially if it became possible to measure polarization in $\mathrm{X}$-ray resonance lines. The radiation of a central source gets strongly polarized upon scattering in a beta-cluster (with a typical resulting degree of polarization $P \sim 60 \%$, Sunyaev 1982), whereas the intrinsic emission of the intracluster gas in X-ray resonance lines is also polarized but to a much lesser degree ( $P \lessgtr 10 \%$, Sazonov et al. 2002). Thus, even a few per cent contribution of scattered AGN radiation to the surface brightness in a resonance line would be manifest in polarimetric observations.

Acknowledgements. We thank the referee, Luca Ciotti, for comments that helped to improve the presentation of the paper. SS acknowledges support from a Peter Gruber Foundation Fellowship. RS as a Gordon Moore Scholar thanks Caltech for hospitality during the completion of this paper. This research was partially supported by the Russian Foundation for Basic Research (projects 00-02-16681 and 0015-96649) and by the program of the Russian Academy of Sciences "Astronomy (Nonstationary astronomical objects)".

\section{References}

Aldrovandi, S. M. V., \& Pequignot, D. 1973, A\&A, 25, 137

Anders, E., \& Grevesse, N. 1989, Geochim. Cosmochim. Acta, 53, 197

Arnaud, M., \& Rothenflug, R. 1985, A\&AS, 60, 425

Arnaud, K. A. 1996, Astronomical Data Analysis Software and Systems V, ed. G. Jacoby, \& J. Barnes, ASP Conf. Ser., 101, 17

Bahcall, J. N., Kirhakos, S., Saxe, D. H., \& Schneider, D. P. 1997, ApJ, 479, 642

Binney, J., \& Tabor, G. 1995, MNRAS, 276, 663

Biretta, J. A., Stern, C. P., \& Harris, D. E. 1991, AJ, 101, 1632

Böhringer, H., Belsole, E., Kennea, J., et al. 2001, A\&A, 365, 181

Boyce, P. J., Disney, M. J., Blades, J. C., et al. 1997, MNRAS, 298, 121

Burderi, L., King, A. R., \& Szuszkiewicz 1998, ApJ, 509, 85

Carilly, C. L., Harris, D. E., Pentericci, L., et al. 2002, ApJ, 567, 781

Cavaliere, A., \& Fusco-Femiano, R. 1976, A\&A, 49, 137

Chandrasekhar, S. 1950, Radiative Transfer (Oxford, Clarendon Press)

Ciotti, L., \& Ostriker, J. P. 1997, ApJ, 487, L105

Ciotti, L., \& Ostriker, J. P. 2001, ApJ, 551, 131

Cramphorn, C. K., \& Sunyaev, R. A. 2002, A\&A, 389, 252

Crawford, C. S., Lehmann, I., Fabian, A. C., Bremer, M. N., \& Hasinger, G. 1999, MNRAS, 308, 1159

Fabian, A. C., Crawford, C. S., Ettori, S., \& Sanders, J. S. 2001, MNRAS, 322, L11

Fang, T. D., Davis, D. S., Lee, J. C., et al. 2002, ApJ, 565, 86

Finoguenov, A., Matsushita, K., Böhringer, H., Ikebe, Y., \& Arnaud, M. 2002, A\&A, 381, 21 
Gilfanov, M. R., Sunyaev, R. A., \& Churazov, E. M. 1987a, Sov. Astron. Lett., 13, 233

Gilfanov, M. R., Sunyaev, R. A., \& Churazov, E. M. 1987b, Sov. Astron. Lett., 13, 3

Hardcastle, M. J., \& Worrall, D. M. 1999, MNRAS, 309, 969

Harris, D. E., Nulsen, P. E. J., Ponman, T. J., et al. 2000, ApJ, 530, L81

Hardcastle, M. J., Birkinshaw, M., \& Worrall, D. M. 2001, ApJ, 323, L17

Hernquist, L. 1989, Nature, 340, 687

Hooper, E. J., Impey, C. D., \& Foltz, C. B. 1997, ApJ, 480, L95

Kaastra, J. S. 1992, An X-Ray Spectral Code for Optically Thin Plasmas (Internal SRON-Leiden Report, updated version 2.0)

Koyama, K., Maeda, Y., Sonobe, T., et al. 1996, PASJ, 48, 249

Lin, D. N. C., \& Shields, G. A. 1986, ApJ, 305, 28

Macchetto, F. D., Marconi, A., Axon, D. J., et al. 1997, ApJ, 489, 579

Marconi, A., Axon, D. J., Macchetto, F. D., et al. 1997, MNRAS, 289, L21

Matsushita, K., Belsole, E., Finoguenov, A., \& Boehringer, H. 2002, A\&A, 386, 77

McLure, R. J., Kukula, M. J., Dunlop, J. S., et al. 1999, MNRAS, 308, 377

McLure, R. J., \& Dunlop, J. S. 2001, MNRAS, 321, 515

Mewe, R., Gronenschild, E. H. B. M., \& van der Oord, G. H. J. 1985, A\&AS, 62, 197

Mineshige, S., \& Shields, G. A. 1990, ApJ, 351, 47

Murakami, H., Koyama, K., \& Maeda, Y. 2001, ApJ, 558, 687

Murphy, B. W., \& Chernoff, D. F. 1993, ApJ, 418, 60

Owen, F. N., Eilek, J. A., \& Kassim, N. E. 2000, ApJ, 362, 449

Rees, M. J. 1988, Nature, 333, 523
Sarazin, C. L., \& Wise, M. W. 1993, ApJ, 411, 55

Sazonov, S. Yu., Churazov, E. M., \& Sunyaev, R. A. 2002, MNRAS, 333, 191

Schade, D. J., Boyle, B. J., \& Letawsky, M. 2000, MNRAS, 315, 498

Shibata, R., Matsushita, K., Yamasaki, N. Y., et al. 2001, ApJ, 549, 228

Sholomitskii, G. B., \& Yaskovich, A. L. 1990, Sov. Astron. Lett., 16, 383

Shull, J. M., \& van Steenberg, M. 1982, ApJS, 48, 95

Siemiginowska, A., Czerny, B., \& Kostyunin, V. 1996, ApJ, 458, 491

Smith, D. A., Wilson, A. S., Arnaud, K. A., Terashima, Y., \& Young, A. J. 2002, ApJ, 565, 195

Sunyaev, R. A. 1982, Sov. Astron. Lett., 8, 175

Sunyaev, R. A., Markevitch, M., \& Pavlinsky, M. 1993, ApJ, 407, 606

Sunyaev, R. A., \& Churazov, E. M. 1998, MNRAS, 297, 1279

Sunyaev, R. A. 1982, Astron. Lett., 8, 175

Verner, D. A., \& Ferland, G. J. 1996, ApJS, 103, 467

Verner, D. A., Verner, E. M., \& Ferland, G. J. 1996, Atomic Data and Nuclear Data Tables, 64, 1

Voronov, G. S. 1997, Atomic Data and Nuclear Data Tables, 65, 1

Wilson, A. S., \& Yang, Y. 2002, ApJ, 568, 133

Wise, M. W., \& Sarazin, C. L. 1990, ApJ, 363, 344

Wise, M. W., \& Sarazin, C. L. 1992, ApJ, 395, 387

Worrall, D. M., Birkinshaw, M., Hardcastle, M. J., \& Lawrence, C. R. 2001, MNRAS, 326, 1127

Yaqoob, T., \& Serlemitsos, P. 2000, ApJ, 544, L95

Young, A. J., Wilson, A. S., Terashima, Y., Arnaud, K. A., \& Smith, D. A. $2002,564,176$

Zombeck, M. V. 1990, Handbook of Astronomy and Astrophysics, second edition (Cambridge, UK: Cambridge University Press) 